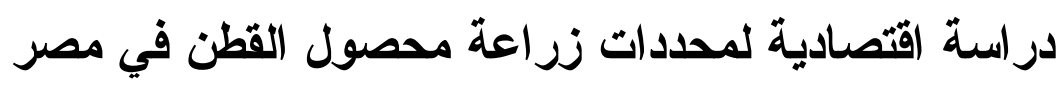

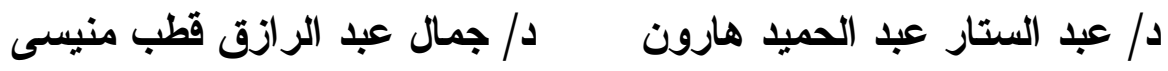

د/ فؤاد محمد حافظ مكي معهد بحوث الاقتصاد الزراعي هارون

مقدمة

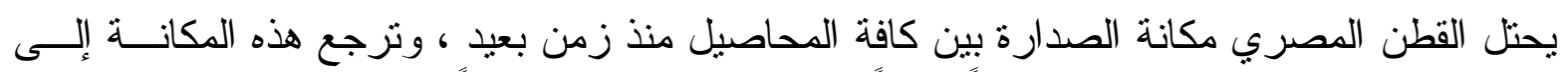

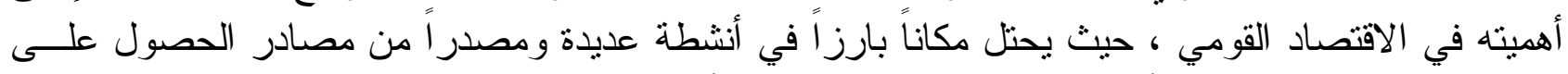

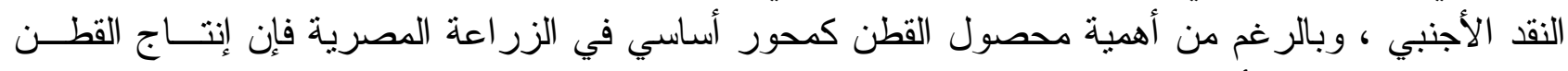

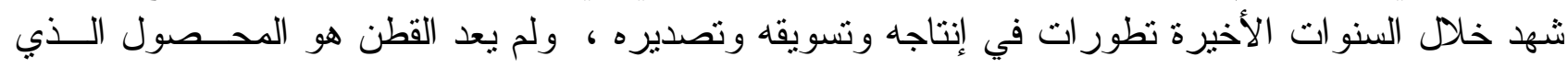

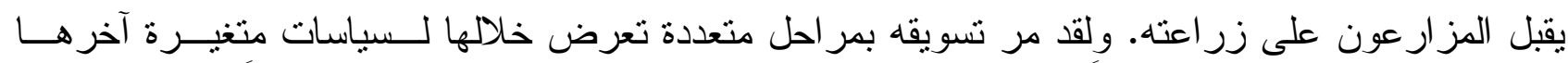

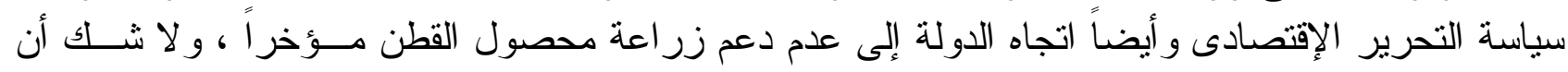

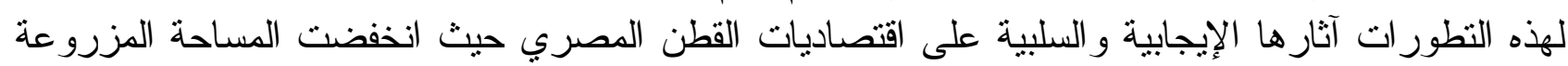

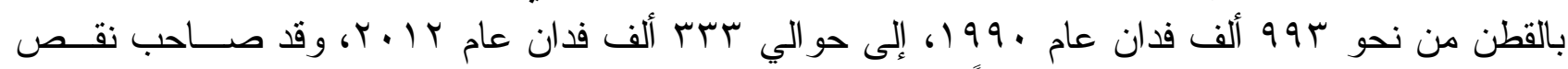

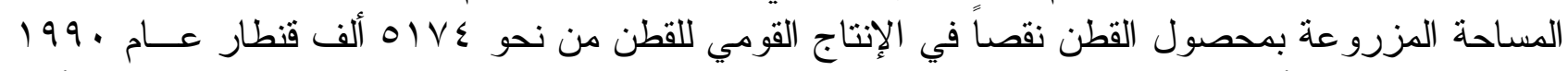

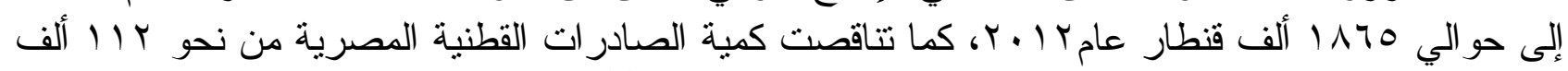

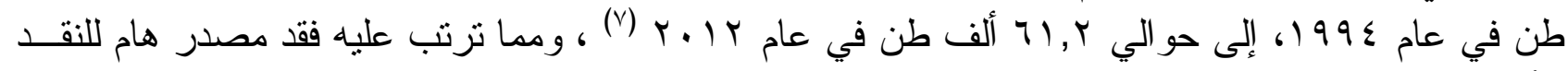

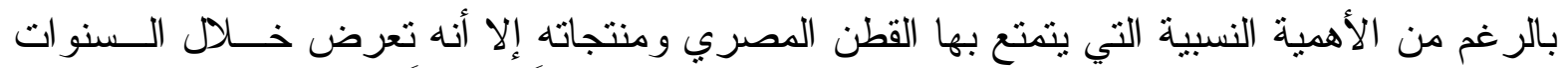

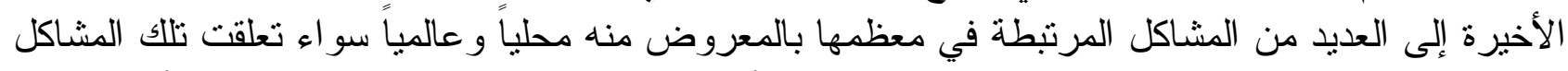

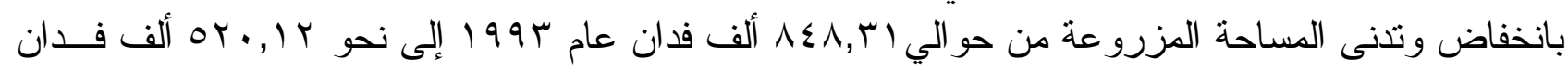

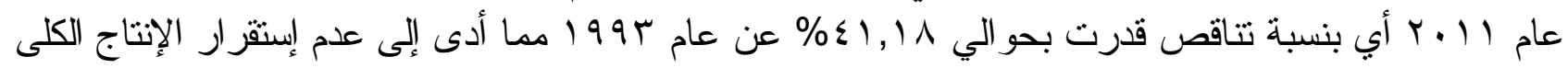

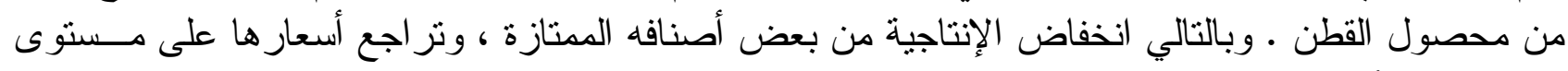

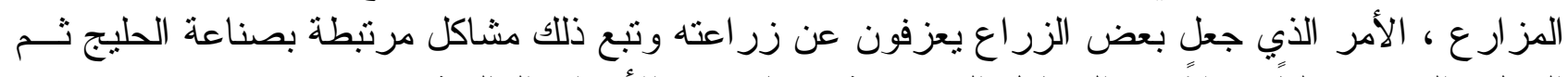

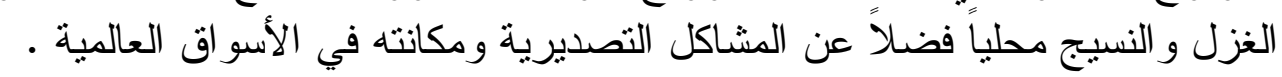

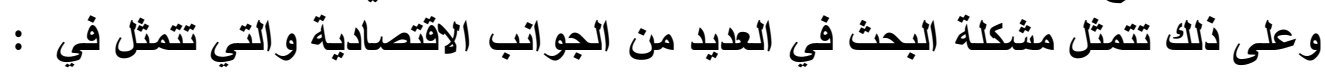

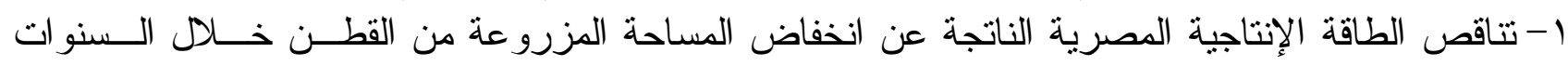

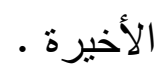

r- تتاقص المعروض القطني من مختلف أصنافه محلياً و عالمياً واللجوء إلى استير اد الغزول.

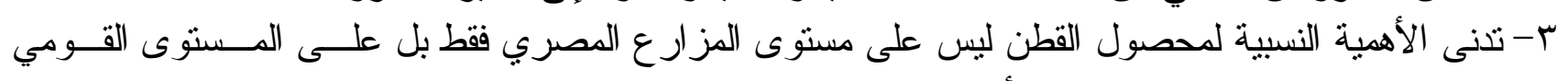

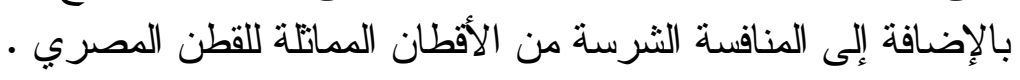

أهداف الار اسة

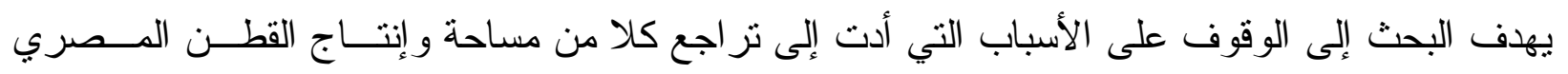

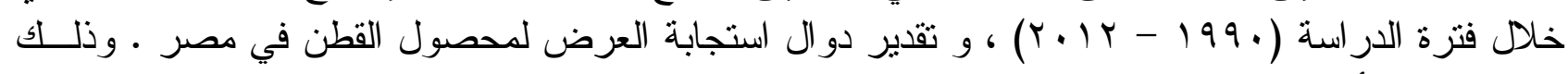

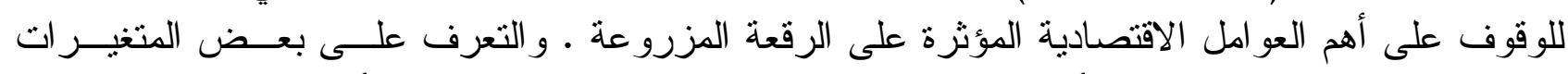

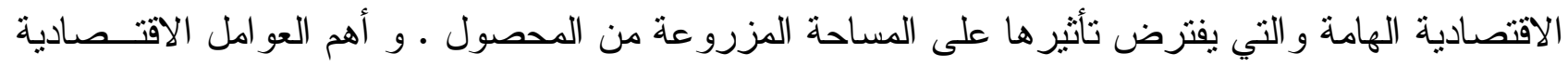

المحددة لإنتاجه .

مصادر البيانات و الطريقة البحثية

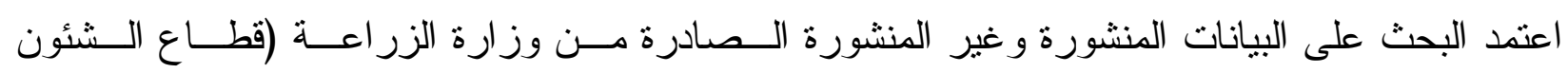

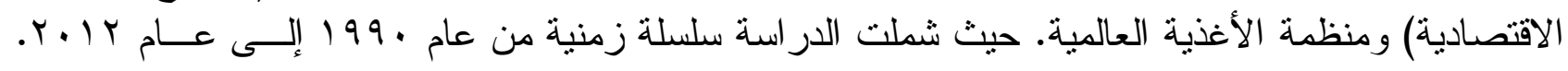




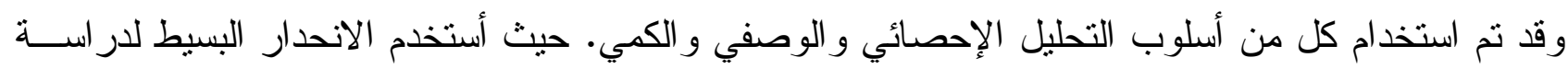

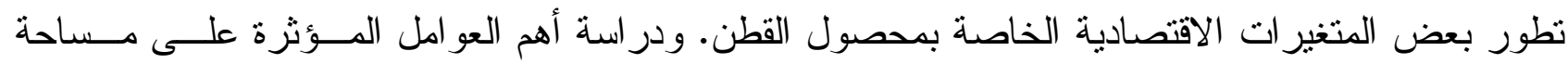
القطن على مستوى الجمهورية أمكن استخدام عدة نماذج فى الصورة الخطية و التي تتضمن مساحة القطن فى ولى ولى

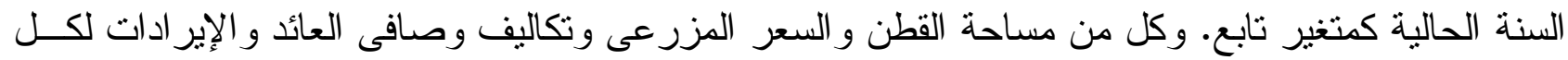

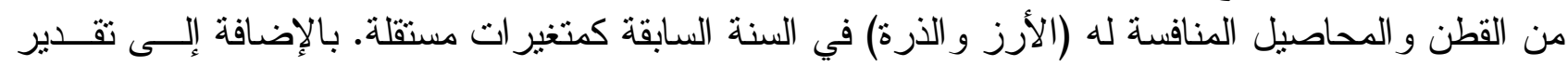

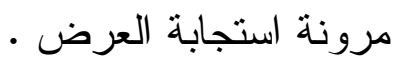

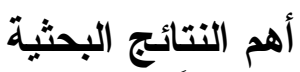

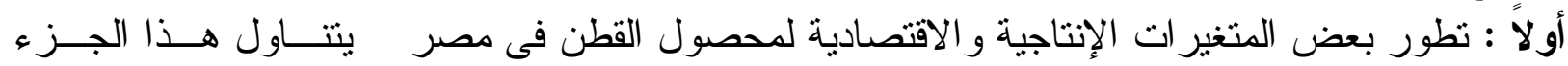

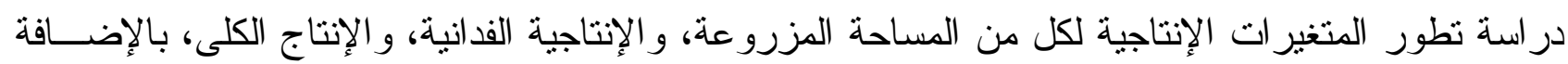

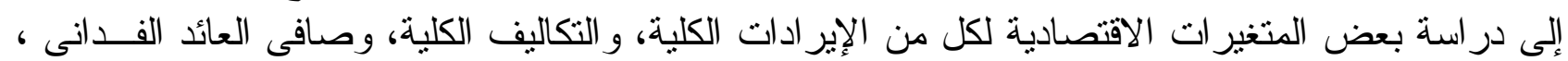

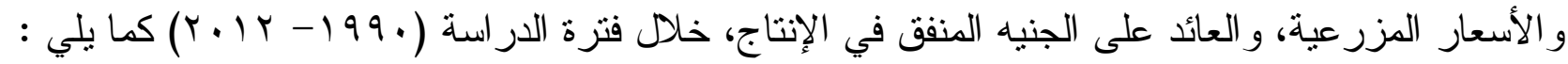

\section{1- تطور مساحة القطن في مصر}

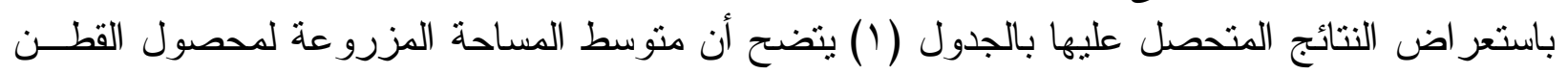

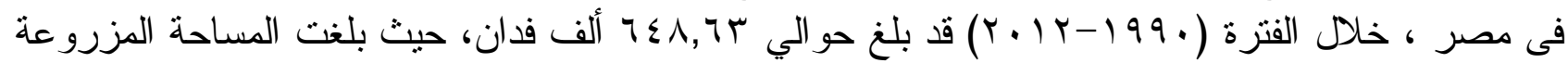

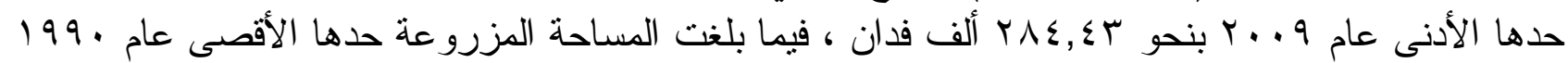

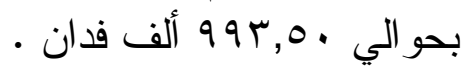

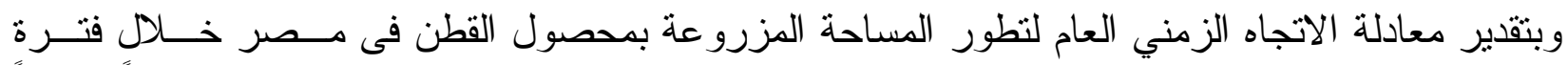

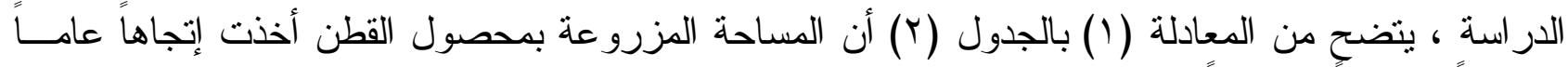

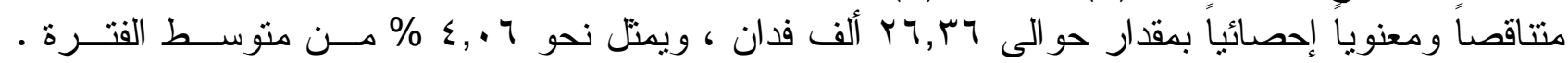

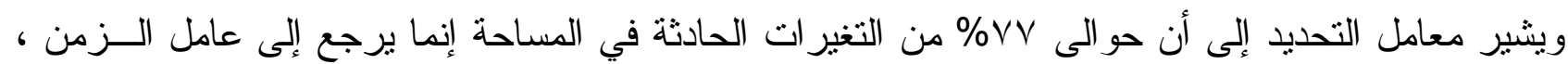

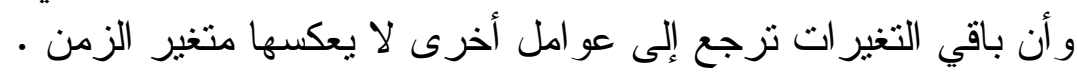

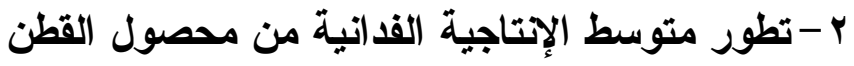

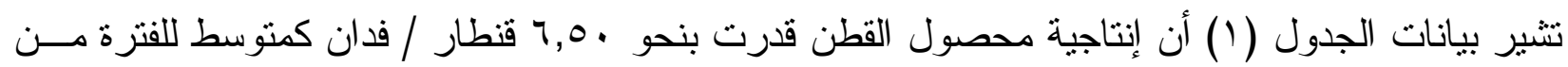

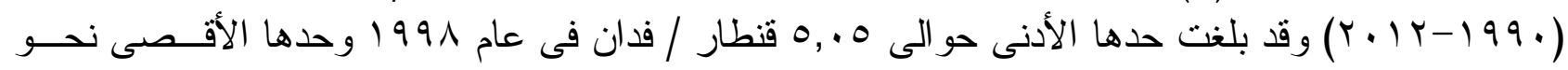

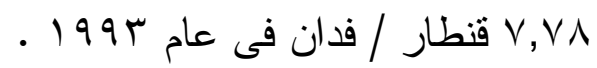
وبتقدير معادلة الاتجاه الزمنى العام يتضح من المعادلة (Y) بالجدول (Y) أن الإنتاجية الفدانية لمحصول

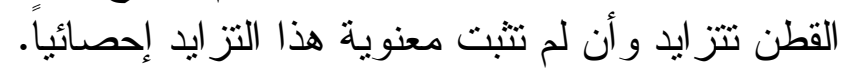
r- تطور الإتتاج الكلى لمحصول القطن

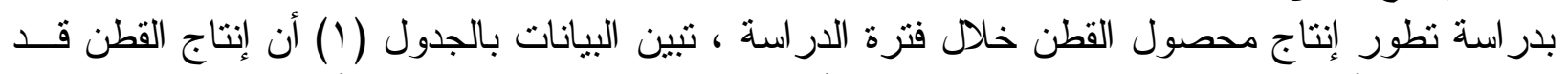

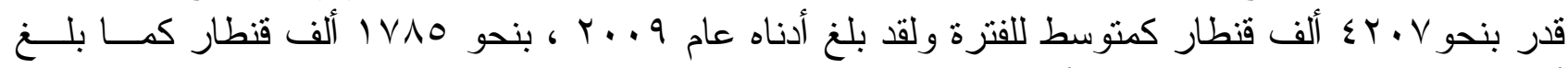

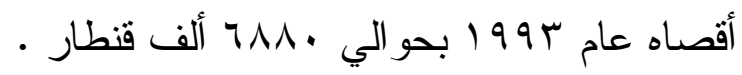

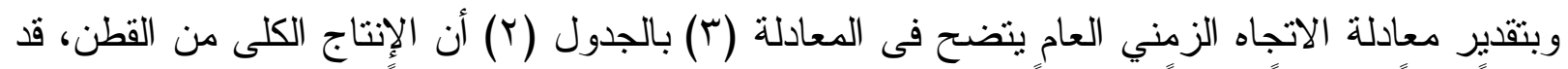

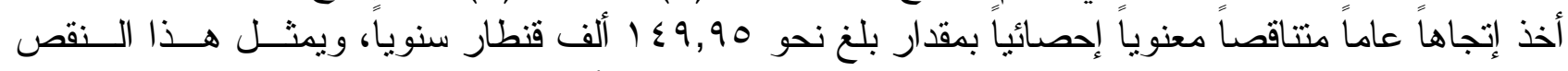

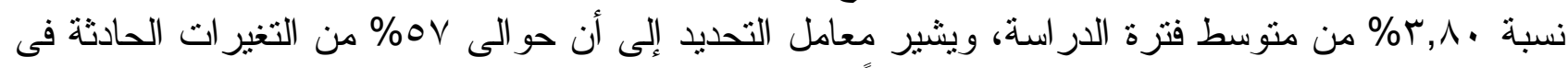

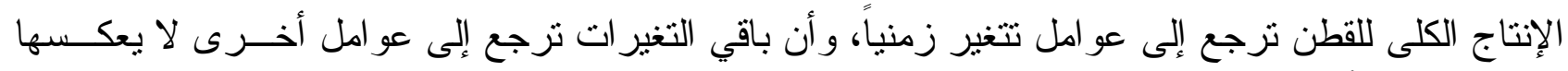

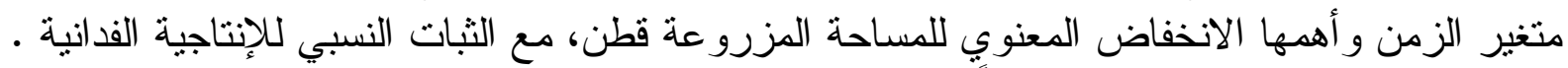

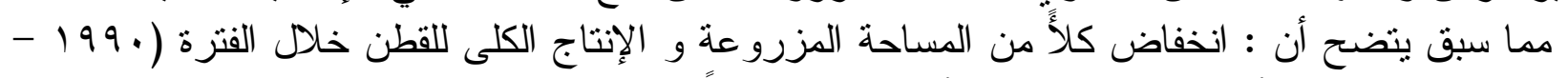

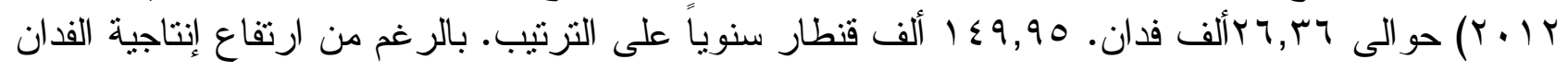
من القطن ارتفاعا طفيفا . 


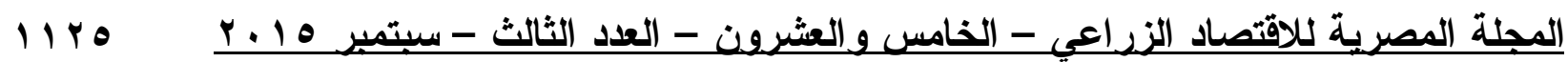

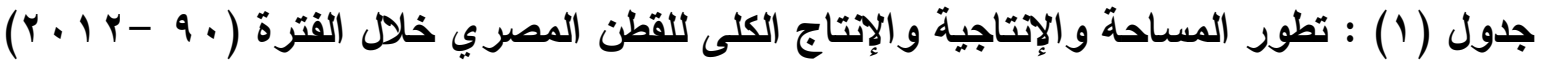

\begin{tabular}{|c|c|c|c|}
\hline (ألإتثاج فنطار & (قنطار / الإذتانة ) & (ألف فاحانة) & السنوات \\
\hline $01 \leqslant V$ & $0, Y 1$ & $994, .0$ & 199. \\
\hline $0 . Y T$ & 0,9 . & $\Lambda 01, Y \Lambda$ & 1991 \\
\hline $7 \cdots 1$ & $V, 10$ & $\overline{\lambda \varepsilon \cdot, r q}$ & 1994 \\
\hline $7 \wedge \Lambda$. & $\overline{V, \vee \Lambda}$ & $\Lambda \wedge \varepsilon, \Gamma)$ & $199 \%$ \\
\hline ETYq & $7, \cdots$ & VYI, $\leqslant \varepsilon$ & $199 \leq$ \\
\hline$\varepsilon .7 Y$ & $0, V Y$ & $V) \cdot, r)$ & 1990 \\
\hline $0 \times 70$ & $7, Y 7$ & $9 r \cdot, 91$ & 1997 \\
\hline $0 \wedge \sum \Gamma$ & $7, \wedge$. & 109,17 & $199 \mathrm{~V}$ \\
\hline T910 & $0, \cdot 0$ & $\nabla \wedge \wedge, \wedge)$ & 1991 \\
\hline rqr. & $7, \cdot V$ & $T \leqslant 0, \leqslant Y$ & 1999 \\
\hline Tolv & $7, \vee \wedge$ & $011, \Gamma Y$ & $r \ldots$ \\
\hline OYAE & $V, Y T$ & VTI,1. & $r \cdots r$ \\
\hline ENTY & 7,10 & $V \cdot 7, \varepsilon)$ & $r \cdots r$ \\
\hline TVTV & $V, \cdot \varepsilon$ & $040, .9$ & $r \ldots r$ \\
\hline 2910 & $7,9 V$ & $V \backslash \varepsilon, V T$ & $r \ldots \varepsilon$ \\
\hline$\varepsilon \cdot 17$ & $7, Y Y$ & 077,01 & $r \ldots o$ \\
\hline rA.q & $v, 1$. & $O V \leqslant, O V$ & $r \cdots r$ \\
\hline एव६. & $7, \wedge\rceil$ & ory, ₹. & $r \cdots v$ \\
\hline T.Y. & $7, \leqslant 7$ & $\Gamma, Y, V A$ & $r \cdots \Lambda$ \\
\hline TVAO & $7, Y \wedge$ & $T \wedge \varepsilon, \varepsilon T$ & $r \ldots q$ \\
\hline TrqV & $7, \leqslant 9$ & rq9, Is & $r \cdot 1$. \\
\hline$\varepsilon \cdot \Gamma \cdot$ & $\mathrm{V}, \mathrm{VO}$ & Or.,IT & $r .11$ \\
\hline 11170 & 0,09 & דיז, & $r \cdot I r$ \\
\hline$\varepsilon r \cdot V$ & 7,0 . & $7 \leqslant \Lambda, 7 \Psi$ & المتوسط \\
\hline
\end{tabular}

المصدر : جمعت وحسبث من

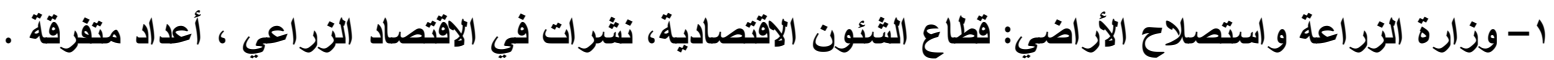

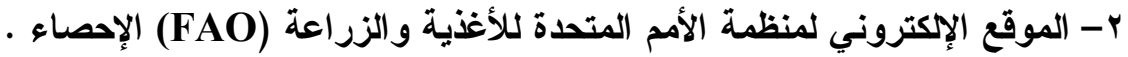
جدول (Y) : نتائج الاتجاه الزمني العام لتطور المساحة المزروعة الامدة والإنتاجية الفدانية والإنتاج الكلى

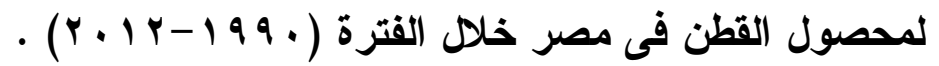

\begin{tabular}{|c|c|c|c|c|c|c|c|}
\hline السنوي التغيز \% & المتوسط & ف & נر & J & المعادلة المقدرة & المتغير التابع & م \\
\hline$\varepsilon, \cdot 7$ & $T \leqslant \Lambda, T r$ & $" * 7,97$ & $\cdot, V V$ & $\cdot, \wedge \wedge$ & 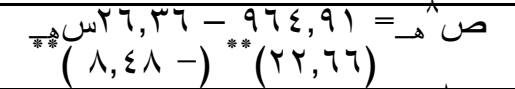 & (بالقطن ألف المزبوان) & (1) \\
\hline 90,01 & $7,0$. & Mr & $\cdot, .0$ & • & 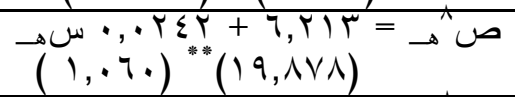 & ألأنتاجية / فذاندانية & $(r)$ \\
\hline$\Gamma, \Lambda$. & $\sum Y \cdot T, V$ & ** YV, Y Y V & $\cdot, 0 \mathrm{~V}$ & $\cdot$, Vo & 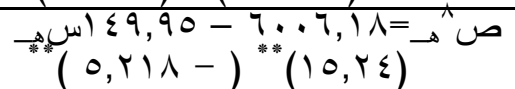 & (ألإنتاج فنطار) & $(r)$ \\
\hline
\end{tabular}

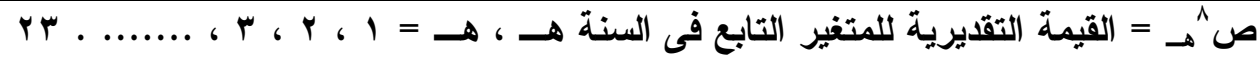

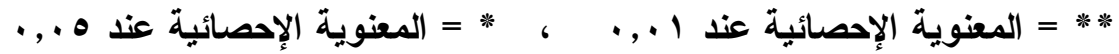

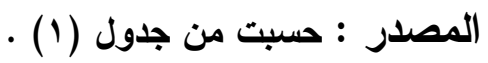
ثانياً : تطور الأهمية النسبية للمساحة المزروعة بمحصول القطن من إجمالي المساحة المحصولية في مصر

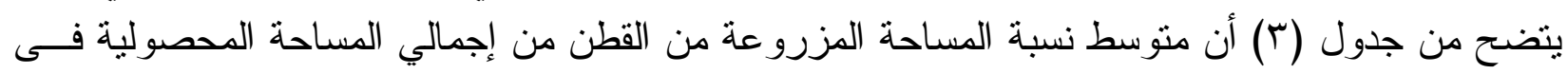

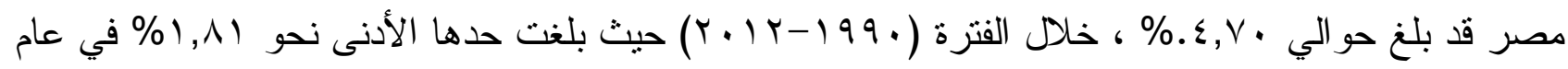

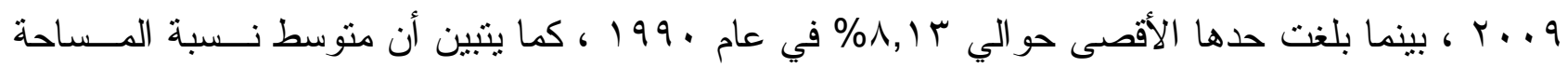

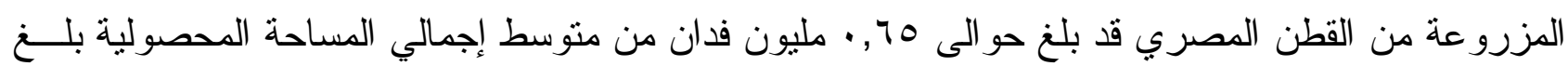

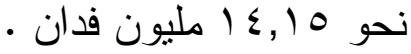


جدول (ب) : تطور نسبة المساحة المزروعة بالقطن من إجمالي المساحة المحصولية في مصر

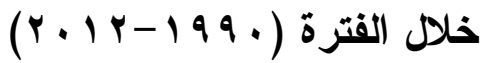

\begin{tabular}{|c|c|c|c|}
\hline نسبة مساحة المحصؤ إليحية إجمائي & (المساحة المحصولية & المساحة المزروبونة بالقطن & لسنوات \\
\hline$\Lambda, \Lambda \Gamma$ & $\mid T, 1 \wedge$ & $\cdot, 99$ & 199. \\
\hline 7,10 & 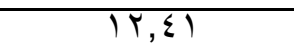 & $\cdot, \wedge 0$ & 1991 \\
\hline $7, V \mu$ & $T_{1}, \varepsilon q$ & $\cdot, \wedge \varepsilon$ & 1994 \\
\hline 7,19 & $I Y, V \wedge$ & $\cdot, \wedge \wedge$ & 1994 \\
\hline $0,0 T$ & $1 \Gamma, \cdot 1$ & $\cdot, V Y$ & 1998 \\
\hline $0,1 \varepsilon$ & $T \Gamma, \Lambda T$ & $\cdot, \mathrm{VI}$ & 1990 \\
\hline 7,11 & $|r, V|$ & $\cdot, 9 Y$ & 1997 \\
\hline $7, Y Y$ & $T \Gamma, \wedge \Gamma$ & $\cdot, \wedge 7$ & $199 \mathrm{~V}$ \\
\hline $0, V$. & $\Pi \Gamma, \wedge\rceil$ & $\cdot, 1 \times 9$ & 1991 \\
\hline$\varepsilon, V T$ & $1 \Gamma, 9 \varepsilon$ & $\cdot, 77$ & 1999 \\
\hline$r, V \varepsilon$ & $1 T, q T$ & $\cdot, 0 Y$ & r... \\
\hline $0, Y$. & $T \varepsilon, \cdot T$ & $\cdot, V T$ & $r \ldots 1$ \\
\hline$\varepsilon, 90$ & $1 \varepsilon, \Gamma_{0}$ & $\cdot, \mathrm{VI}$ & $r \ldots r$ \\
\hline$r, V T$ & $1 \varepsilon, \varepsilon V$ & $\cdot, 0 \leqslant$ & $r \ldots r$ \\
\hline$\varepsilon, \wedge \wedge$ & $1 \leqslant, 00$ & $\cdot, \mathrm{VI}$ & $r \ldots \varepsilon$ \\
\hline$r, \wedge Y$ & $|\varepsilon, 9|$ & $\cdot, 0 \mathrm{~V}$ & r...o \\
\hline$r, \wedge T$ & $1 \varepsilon, 9 T$ & $\cdot, 0 \mathrm{~V}$ & $r \ldots T$ \\
\hline$r, 07$ & 10,11 & $\cdot, 0 \leqslant$ & $r \ldots V$ \\
\hline$T, \cdot T$ & $10, Y \varepsilon$ & $\cdot, \Gamma$ & $r \ldots \Lambda$ \\
\hline $1, \lambda 1$ & $10,0$. & $\cdot, \Gamma \wedge$ & $r \ldots q$ \\
\hline$r, \xi)$ & 10,r & $\cdot, \Gamma V$ & $Y .1$. \\
\hline$r, r q$ & $10, r_{0}$ & $\cdot, O Y$ & $r . \mid l$ \\
\hline$T, I T$ & 10,07 & • & Y.IY \\
\hline$\varepsilon, \Gamma_{0}$ & $1 \varepsilon, 10$ & $\cdot, 70$ & لمتوسط \\
\hline
\end{tabular}

المصدر : جمعت وحسبت من وزارة الزراعة واستصلاح الأراضي : قطاع الثئون الاقتصادية ، نشرات الإحصاء الزراعي،

ثالثاً: تطور الأهمية النسبية للإنتاج المحلى للقطن المصرى مقارنة بنظيره العالمي

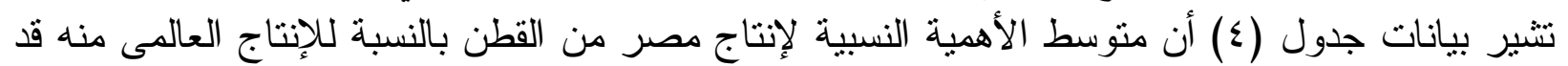

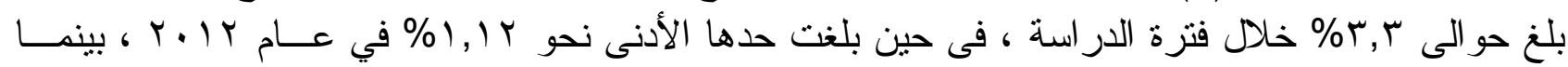

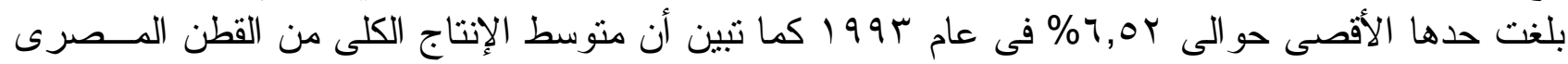

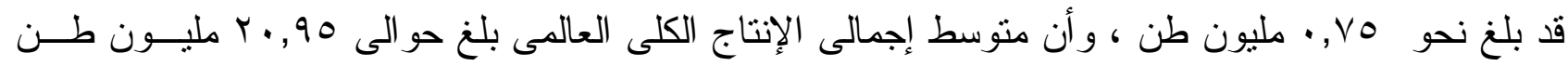

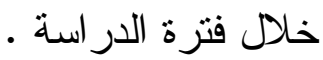
رابعاً: تطور الإير ادات والتكاليف الفدانية وصافى العائد الفدانى والسعر المزرعى والجنيه المنفق لمحصول القطن ا- تطور الإير ادات الكلية للفدان من محصول القطن

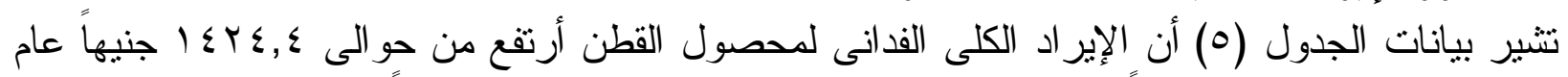

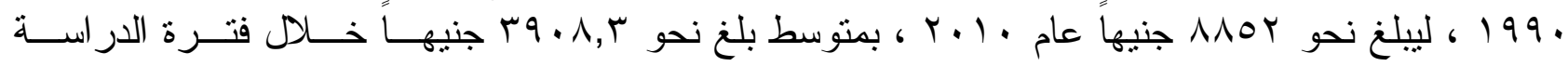
$\cdot(r \cdot 1 r-199 \cdot)$

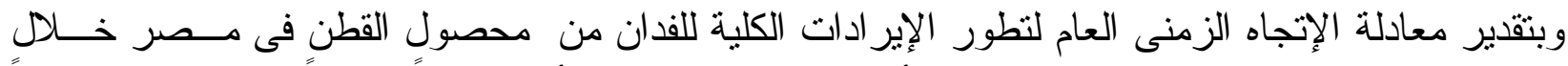

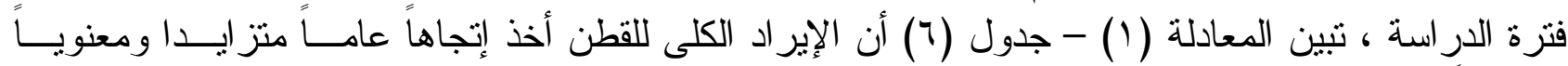

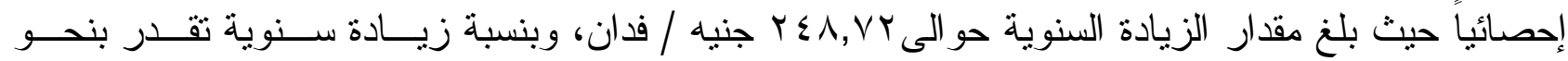

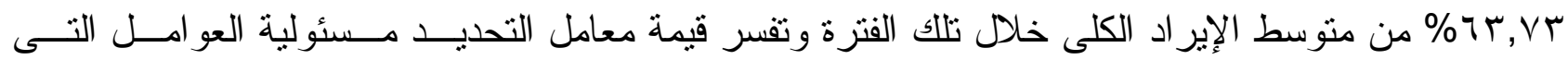




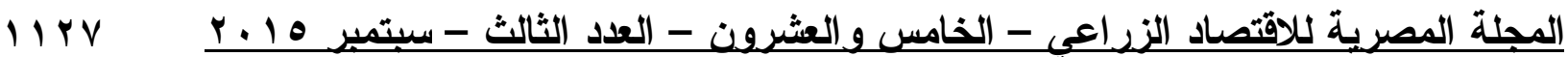

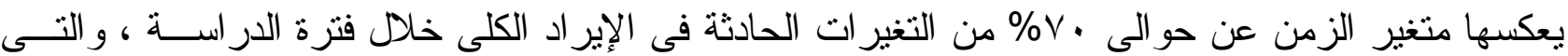

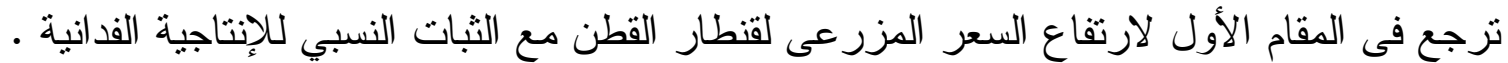

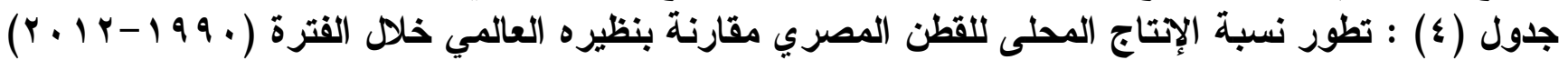

\begin{tabular}{|c|c|c|c|}
\hline \multicolumn{3}{|c|}{ الإنتاج الكلى ( مليون طن } & \multirow{2}{*}{ السنوات } \\
\hline \% مصر للعالم & في العُالم & في مصر & \\
\hline$\varepsilon, T V$ & T1,0T & $\cdot, \Lambda 1$ & 199. \\
\hline$T, \Lambda T$ & $r \cdot, 79$ & $\cdot, \mathrm{V} 9$ & 1991 \\
\hline 0,11 & 11,17 & $\cdot, 9 \xi$ & 1998 \\
\hline $7,0 Y$ & 17,07 & $1, \cdot 1$ & 1994 \\
\hline$\Gamma, V \cdot$ & 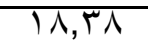 & $\cdot, 71$ & $199 \varepsilon$ \\
\hline$T, Y V$ & 19,7 & $\cdot, 7 \xi$ & 1990 \\
\hline$\xi, V Y$ & 19,19 & $\cdot, 91$ & 1999 \\
\hline$\xi, \Lambda \Gamma$ & $19, \cdot \varepsilon$ & $\cdot, 97$ & $199 \mathrm{~V}$ \\
\hline$\Gamma, \varepsilon V$ & 11,17 & $\cdot, 7 \pi$ & 1991 \\
\hline$r, \xi 1$ & $1 \Lambda, Y \cdot$ & $\cdot, 7 T$ & 1999 \\
\hline T,9V & $|1,0|$ & $\cdot, 00$ & $r \cdots$ \\
\hline$\Gamma, 9 \leqslant$ & $T 1, \cdot V$ & $\cdot, \Lambda \Gamma$ & $Y \cdots 1$ \\
\hline$\varepsilon, \cdot 1$ & 11,19 & $\cdot, \mathrm{V} \mathrm{V}$ & $r \ldots r$ \\
\hline$r, \cdot \Lambda$ & $19, \Sigma V$ & $\cdot, 7$ & $r \cdots r$ \\
\hline$T, Y, T$ & TE,OT & $\cdot, \mathrm{V}^{9}$ & Y.. \\
\hline$r, \cdot T$ & $T \leqslant, \varepsilon V$ & $\cdot, \mathrm{V} \varepsilon$ & Y..O \\
\hline T, , $\varepsilon$ & T $\leqslant, 0$. & $\cdot, \mathrm{V} Y$ & P.. \\
\hline T,97 & $T_{0, \cdot \varepsilon}$ & $\cdot, \mathrm{V} \varepsilon$ & $Y \cdots V$ \\
\hline$\Gamma, \Gamma \wedge$ & TY, $\leqslant T$ & $\cdot, V^{7}$ & $r \cdots \Lambda$ \\
\hline$\Gamma, 79$ & $T \cdot, \lambda V$ & $\cdot, \mathrm{V} \mathrm{V}$ & P.. q \\
\hline$\Gamma, T$ & $T T, 07$ & $\cdot, \mathrm{V} \wedge$ & $Y \cdot 1 \cdot$ \\
\hline$\Gamma, \cdot \varepsilon$ & To,90 & $\cdot, \mathrm{V9}$ & $Y \cdot 11$ \\
\hline $1,1 Y$ & 10,97 & $\cdot, 19$ & $r \cdot T r$ \\
\hline$\Gamma, \Gamma$ & $T \cdot, 90$ & $\cdot, \mathrm{VO}$ & آلمتوسط \\
\hline
\end{tabular}

المصدر : جمت وحسبت من

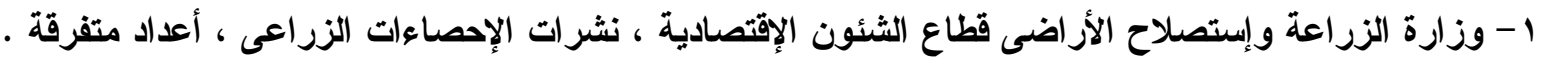

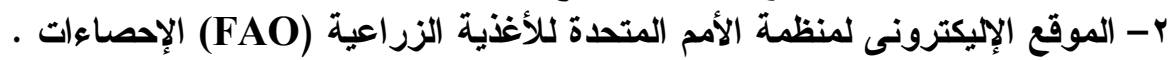

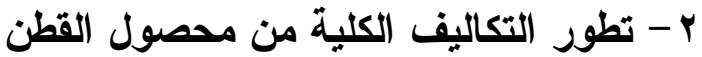

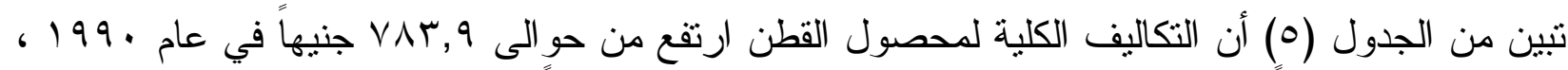

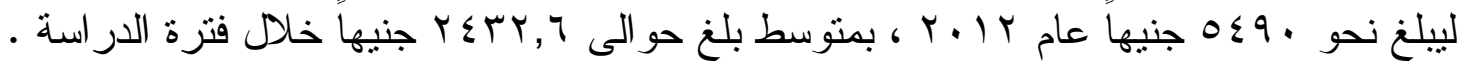

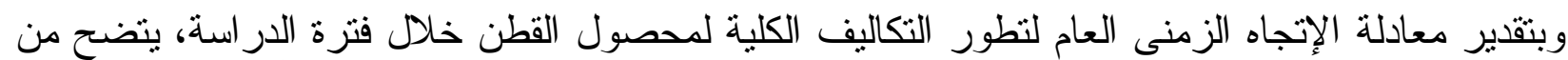

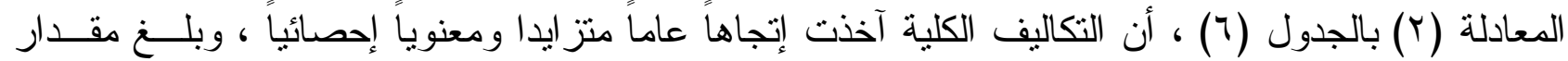

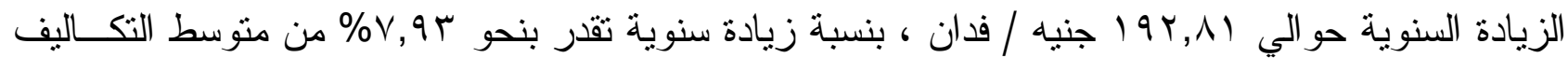

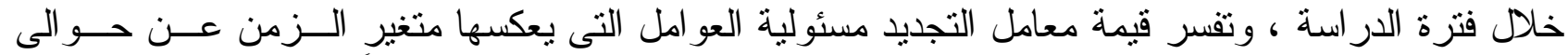

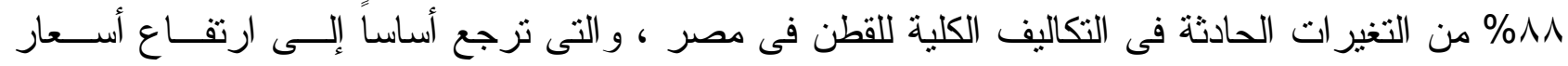

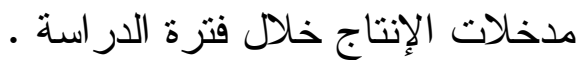

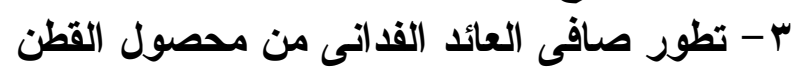

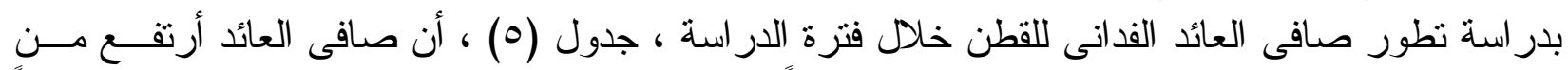

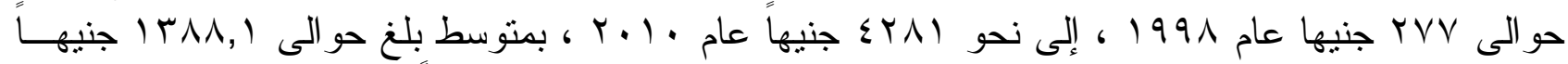

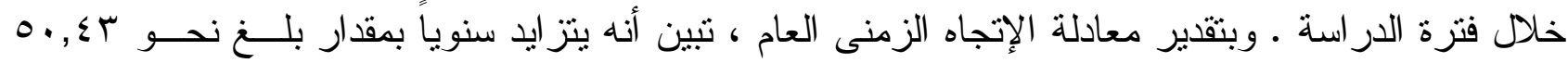

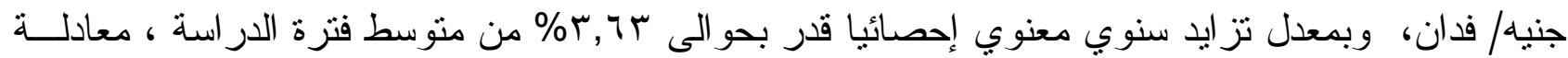

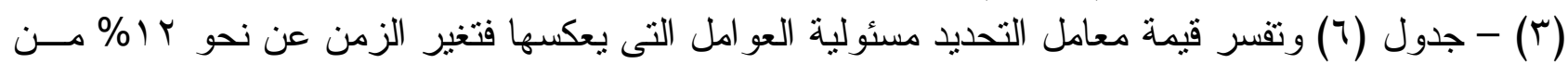

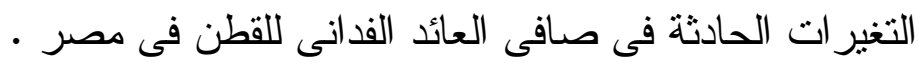


ع - تطور السعر المزرعى لمحصول القطن

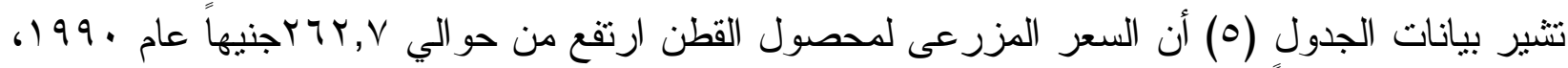

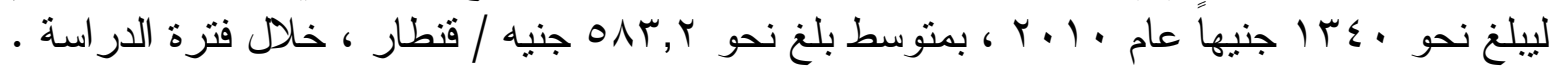

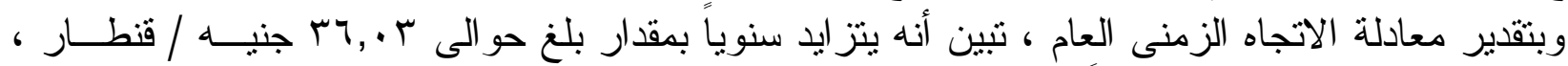

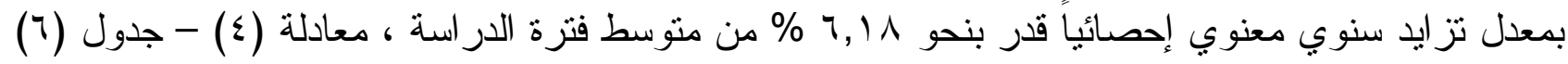

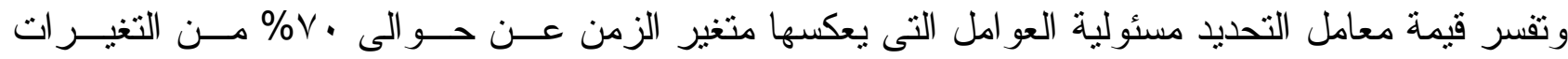

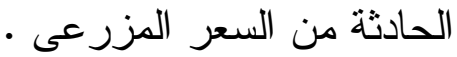

جدول (•) : تطور الإيرادات والتكاليف الكلية وصافى العائد الفدانى والسعر المزرعى الجنيه المنفق

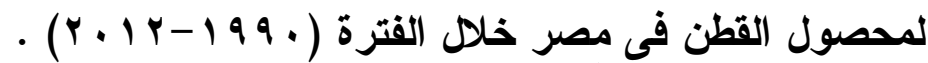

\begin{tabular}{|c|c|c|c|c|c|}
\hline أربحية الجنيه المنفق & ألسعر/المزارعى & صافى الكعائد الفـأنى & ألتكاليف:فانية) الكلية & الإير اديه) الكلى & السنوات \\
\hline$\cdot, \lambda Y$ & $T A T, V$. & $7 \leqslant \cdot, 0\}$ & $\nabla \wedge T, q$ & 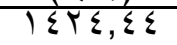 & 199. \\
\hline 1,19 & T17,7. & $1 \cdot \sum q, r q$ & Avq, & $19 Y \wedge, r 4$ & 1991 \\
\hline $1, \mathrm{VA}$ & $T V V, V T$ & $T \vee \vee \wedge, 1 \cdot$ & $911, Y$. & TV०9,r. & 1994 \\
\hline $1,1 \pi$ & $T V, 1 T$ & TOTr,V. & Tारл, Y & Tqहᄉ, q. & 1994 \\
\hline$\cdot, \varepsilon \leqslant$ & TYO,T & $71, Y$. & $1 \varepsilon \cdots, \varepsilon$ & $r \cdot 11,7$. & $199 \varepsilon$ \\
\hline 1,19 & $0 \leqslant \Gamma, \vee 0$ & $1 V र 9,0$. & $T \leqslant 0 \cdot, \cdot$ & TाV9,0. & 1990 \\
\hline 1,71 & $011, \varepsilon$. & 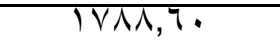 & $1 \cdot 7 T, Y$ & TYVq,q. & 1997 \\
\hline $1, \cdot \Gamma$ & $\varepsilon V T, \cdots$ & TIVT,1. & $17 \times 7,1$ & rTqA, r. & $199 \mathrm{~V}$ \\
\hline$\cdot, 11$ & $T \leqslant \wedge, q$. & $T V V, \ldots$ & 1070,1 & 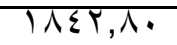 & 1991 \\
\hline$\cdot, 11$ & $r \leqslant 9, \cdots$ & TTE,7. & TAET,V & TाVA,r. & 1999 \\
\hline$\cdot, 19$ & To., 1. & 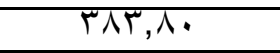 & T.OT,q & TETन,V. & Y... \\
\hline$\cdot$, YO & $\Gamma \leqslant \vee, \ldots$ & $01 \cdot, V$. & $T \cdot 7 \Lambda, Y$ & TOVA, 9. & $r \cdots 1$ \\
\hline$\cdot, \sum$ (ा) & $\sum 1,, \ldots$ & $\Lambda \varepsilon \varepsilon, \cdots$ & $T \cdot 7 T, \cdot$ & $r q \cdot v, \cdots$ & $r \ldots r$ \\
\hline$\cdot, \Lambda \Gamma$ & $010, \ldots$ & TVO\&,... & Tा1,. & r人70,.. & $r \cdots r$ \\
\hline$\cdot, 95$ & $710, \ldots$ & Yार, . & TYVO,. & $\varepsilon r q T, \cdots$ & $r \cdots \varepsilon$ \\
\hline$\cdot, \mathrm{V} 9$ & $V 74, \cdots$ & T.OA, . & YचाV, & rTVo,.. & $r \ldots o$ \\
\hline$\cdot, 91$ & $\nabla \wedge \cdot, \cdots$ & rq19, ,. & Y970,. & $070 \xi, \ldots$ & $r \ldots 7$ \\
\hline$\cdot, \mathrm{T \Lambda}$ & $7 V 1, \cdots$ & Tr99,.. & $T \varepsilon \Gamma, \cdot$ & $\varepsilon V \Gamma 7, \cdots$ & $r \cdots v$ \\
\hline$\cdot, \Gamma$ & $\Lambda \cdot 7, \cdots$ & TYTV,.. & $\varepsilon \mid{ }^{\prime}, \cdot \cdot$ & Or $\leqslant \vee, .$. & $r \ldots 1$ \\
\hline$\cdot, 1$. & $7 V 9, \ldots$ & $\varepsilon \cdot \Gamma, \cdots$ & ए991,. & $\varepsilon \varepsilon, 1, \cdots$ & $p \ldots 9$ \\
\hline$\cdot, 9 \varepsilon$ & $\pi \leqslant \varepsilon, \cdots$ & $\sum Y \wedge \mid, \cdots$ & हOVI, & 1イ०Y,.. & $r .1$. \\
\hline$\cdot, \pi T$ & $1.77, \cdots$ & Trlo,.. & $0194,$. & $\Lambda \Sigma \Lambda \cdot, \cdots$ & $r .11$ \\
\hline$\cdot, Y Y$ & $1179, \ldots$ & TYTR,.. & $0 \leqslant 9 \cdot, \cdot$ & TVIT,... & $r+1 Y$ \\
\hline$\cdot, V Y$ & $O \wedge T, Y$ & $T \Gamma \wedge \Lambda, 1$ & $T \leq T, T$ & $r q \cdot 1, r$ & المتوسط \\
\hline
\end{tabular}

المصدر : جمعت وحسبت من : وزارة الزراعة وإستصلاح الأراضى : قطاع الشئون الإقتصادية ، نـشرات الإحسـصاءات

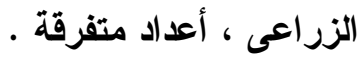

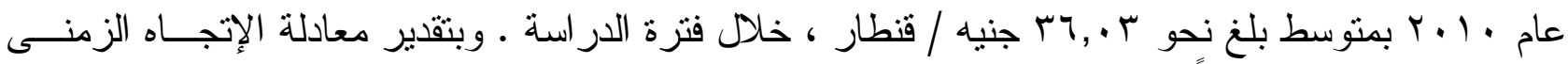

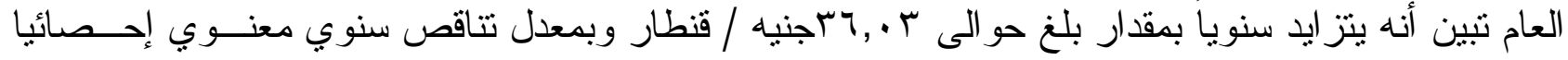

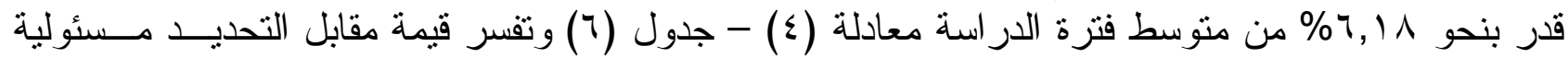

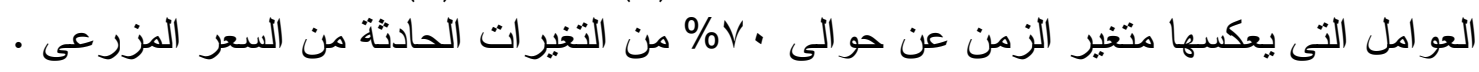

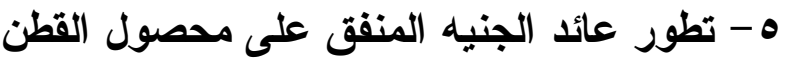

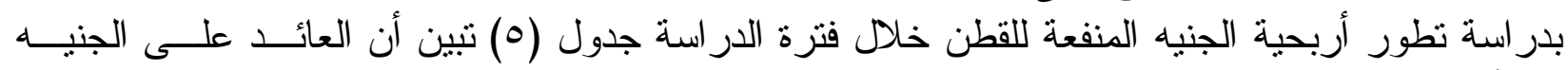

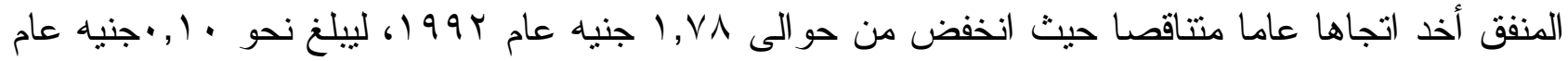

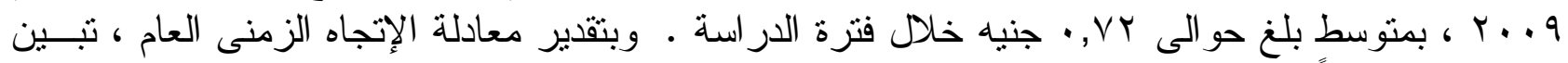

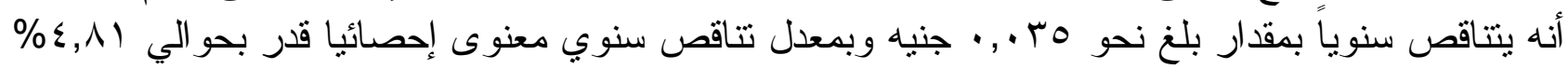

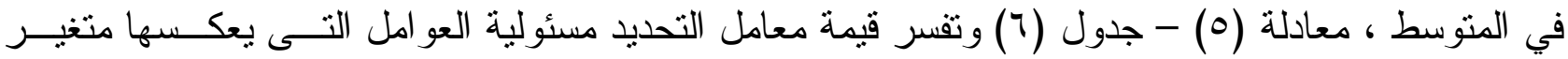

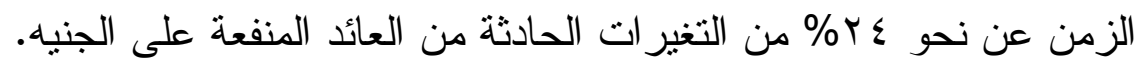


المجلة المصرية للاقتصاد الزراعي - الخامس والعشرون - العدد الثالث - سبتمبر 10 ب

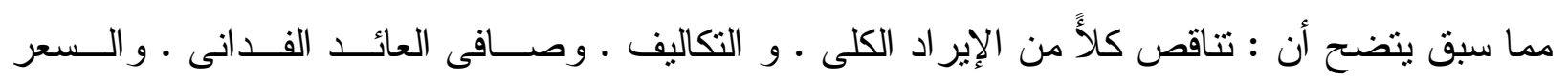

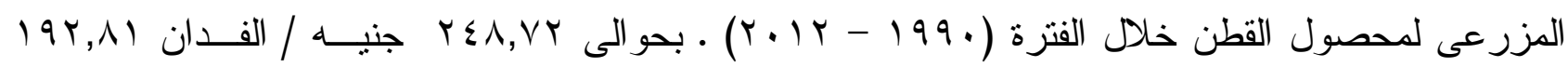

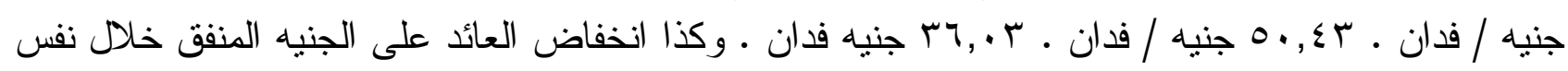

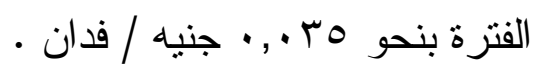

جدول ( ا) : تقديرات الإتجاه الزمنى العام لتطور الإير ادات الكلية و التكاليف وصافى العائد والسعر

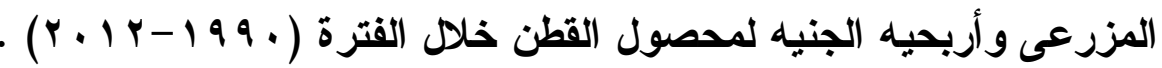

\begin{tabular}{|c|c|c|c|c|c|c|c|}
\hline السنويز & المتوسط & ف & ر & J & المعادلة المقدرة & المتغير التابع & b \\
\hline Tr,VT & $r q \cdot \wedge, r$ & $* * \leqslant \wedge, \wedge \vee q$ & $\cdot, \vee \cdot$ & $\cdot, \wedge \varepsilon$ & 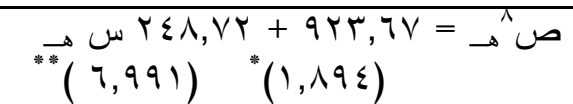 & الإيراد الكلى (جنيه) & (1) \\
\hline$\vee, q \mu$ & $T \leqslant T r, T$ & $* 107,70$ & $\cdot, \wedge \wedge$ & $\cdot, 9 \leq$ & 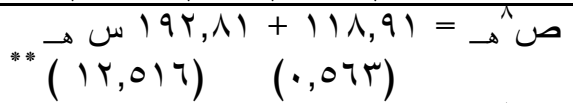 & التكاليف الكلية & $(r)$ \\
\hline ז זד, & 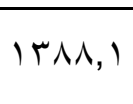 & "Y, & $\cdot, 1 T$ & • & 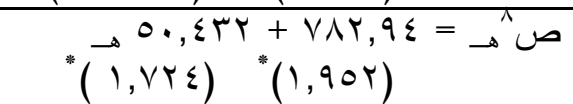 & الفدانى (جنيه) & $(r)$ \\
\hline 7,11 & ONr, r & $* * \wedge . . . \wedge$ & $\cdot, V \cdot$ & $\cdot, \lambda \Gamma$ & 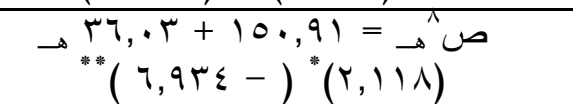 & (السعز المزرعى (جنطار) & $(\varepsilon)$ \\
\hline$\varepsilon, \wedge)$ & $\cdot, V Y$ & $" 7$, or & $\cdot, r \leq$ & $\leqslant 9 \ldots$ & 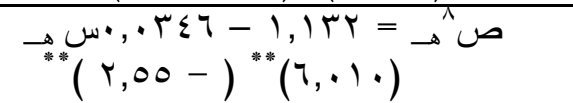 & أربحية الجنيه & $(\theta)$ \\
\hline
\end{tabular}

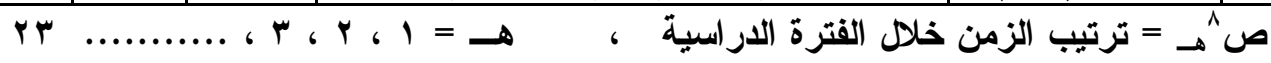

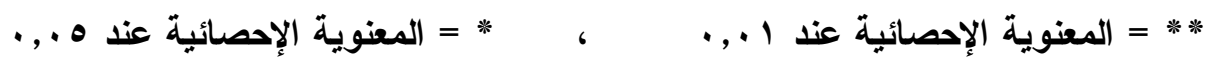

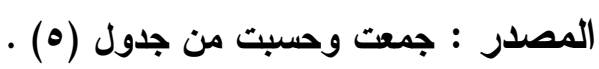

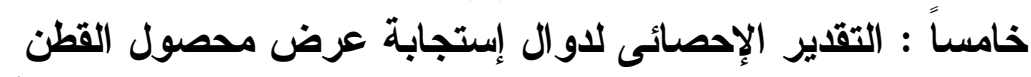

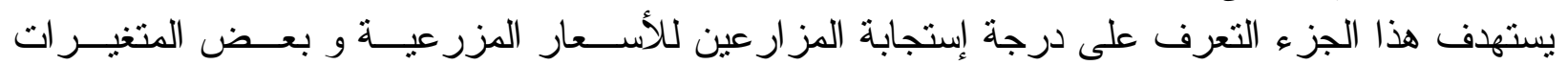

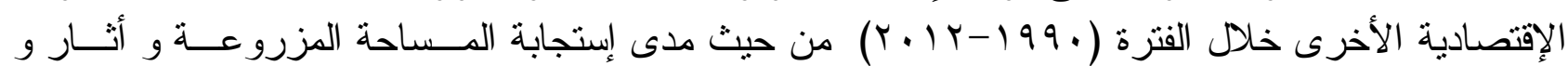

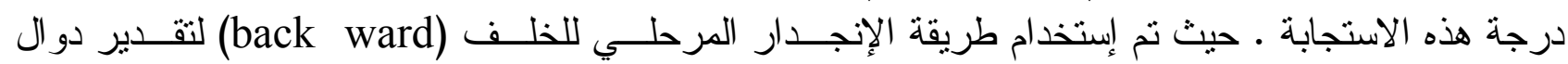

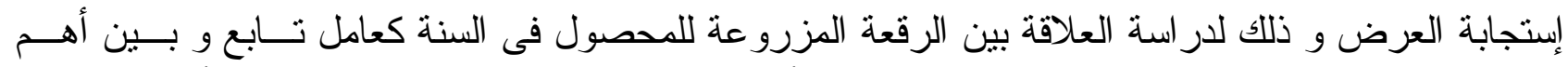

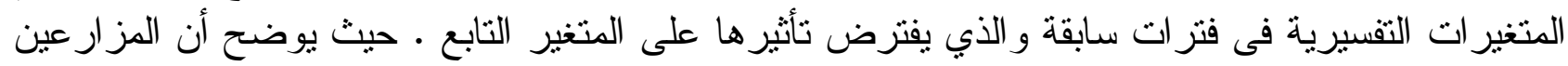

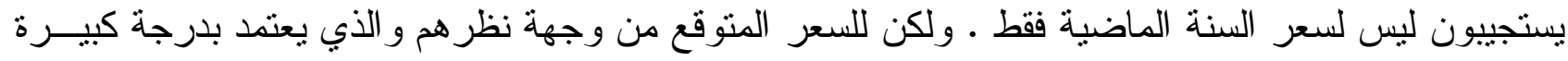

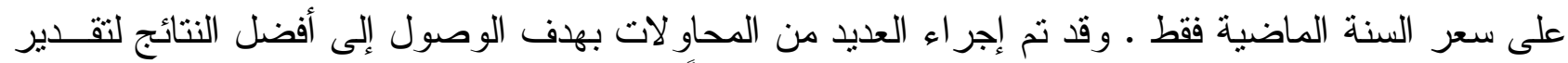

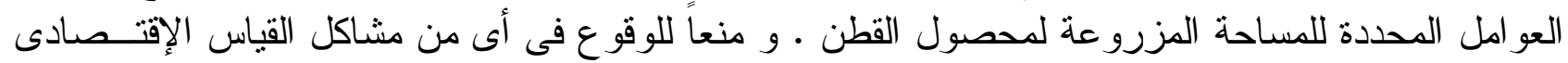

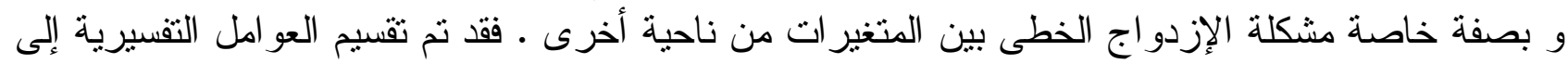

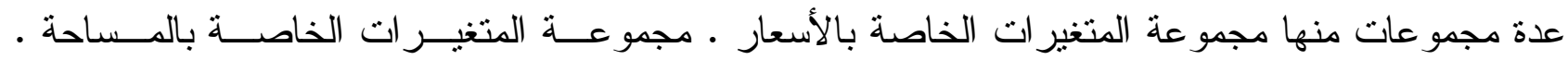

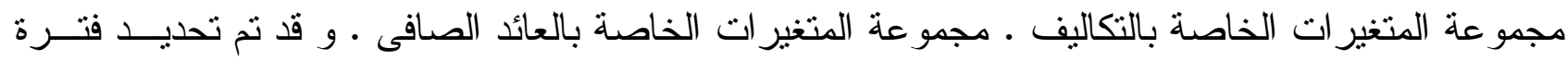

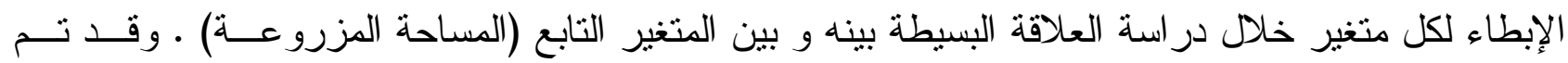

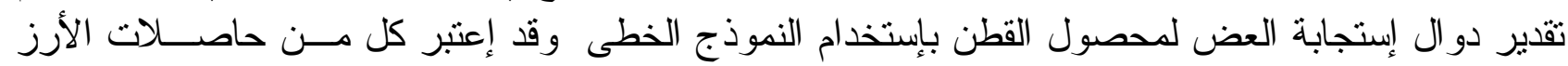

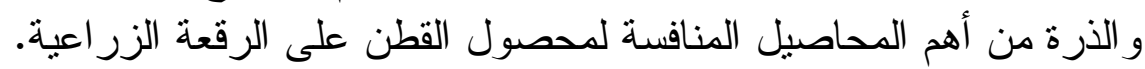

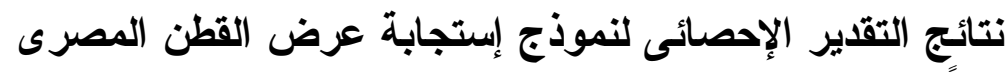

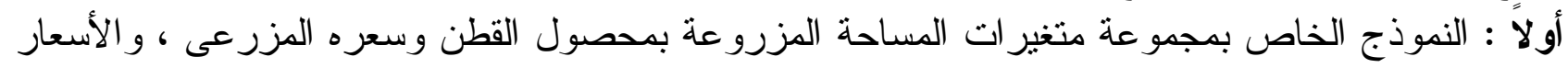

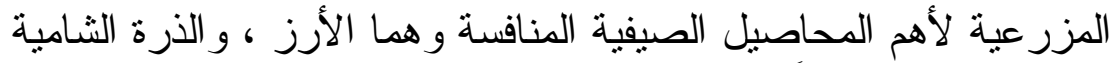
وقد تبين أن أكثر ها تمشياً لطبيعة البيانات و أدقها نتائج إحصائية و إقتصاية الصورة الخدة الخطية وهى كالتـالتي : الصورة العامة للمعادلة : 


$$
\begin{aligned}
& \text { ص ^د = }
\end{aligned}
$$

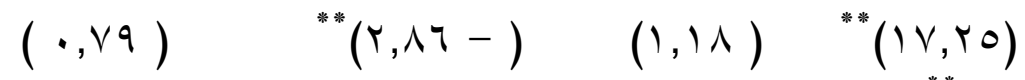

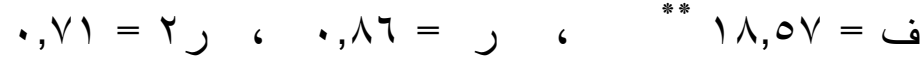

حيث : ص هـ = مساحة القطن فى السنة الحالية (هـ) بالألف فدان .

سا : السعر المزرعى لقنطار القطن فى السنة الحالية بالجنيه .

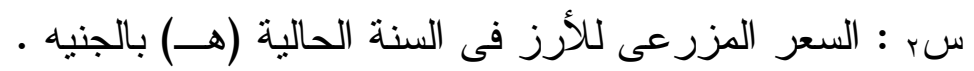

سr : السعر المزرعى للذرة الثامية فى السنة الحالية (هـ) بالجنيه

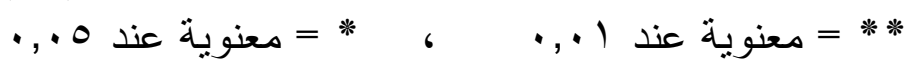

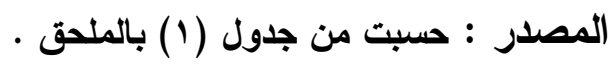

تشير الصورة العامة من البيانات الو اردة بجدول رقم (1) بالملحق الذي يبين العلاقة الدالية بـين مـسـاحة

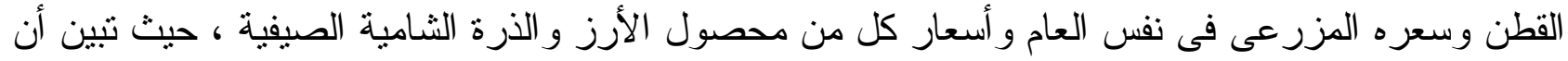
هنالك علاقة طردية بين مساحة القطن وسعره المزرعى ، وهذا يتفق مع المنطق الإقتصادى ، إلا إنه لم تثبت

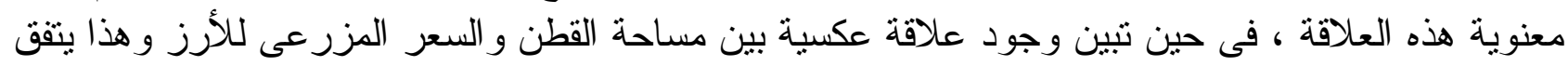

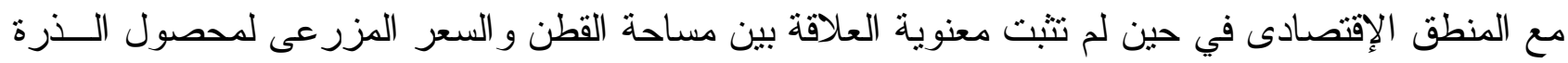
الثامية ـ الثعاية

الصورة النهائية للمعادلة :

$$
\begin{aligned}
& \text { ص מA } \\
& \text { *** }(V, Y \cdot-)^{* *}(19, r \cdot) \\
& \text { •, } \vee=r J \quad \text { • }
\end{aligned}
$$

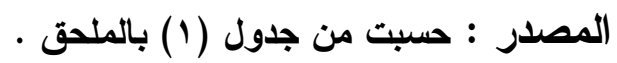

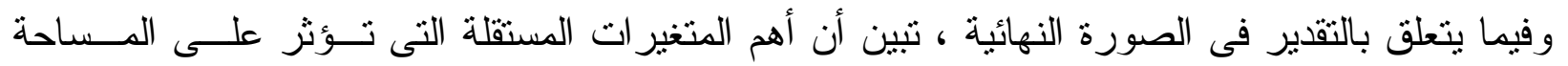

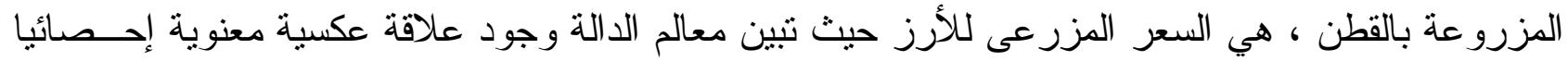

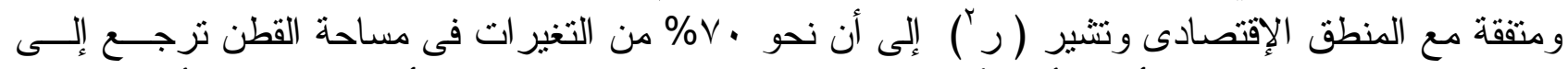

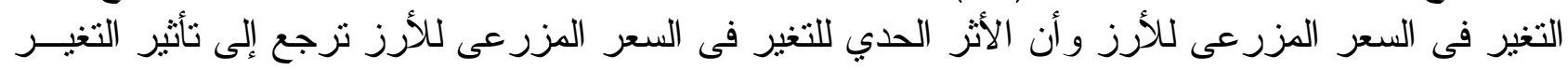

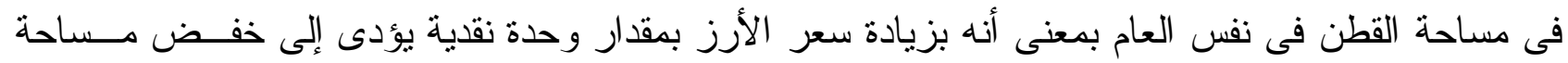

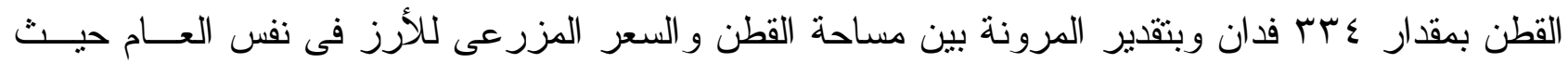

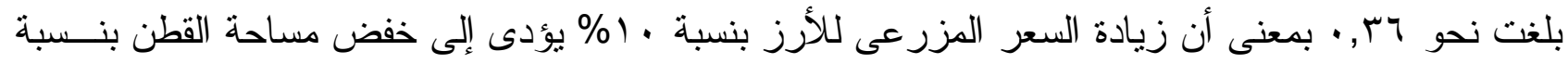

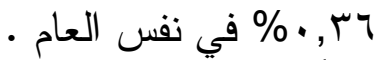

ثاتياً : العلاقةة بين مساحة القطن والسعر المزرعى للقطن والسعر المزرعى للأرز والسعر المزرعى للــذرة الشامية للسنة السابقة

الصورة العامة (وهى النهائية) :

$$
\begin{aligned}
& \text { ص ^ه }
\end{aligned}
$$

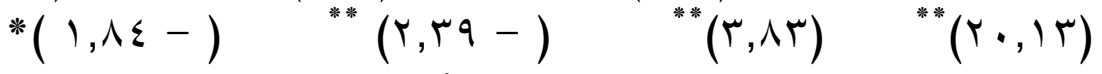

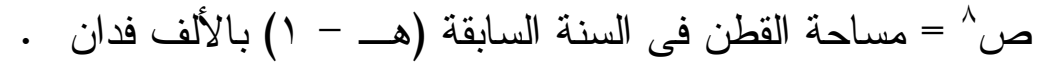

$$
\begin{aligned}
& \text { س = السعر المزرعى للقطن فى السنة السابقة (هـ - ( ) جنيه / قنطار }
\end{aligned}
$$

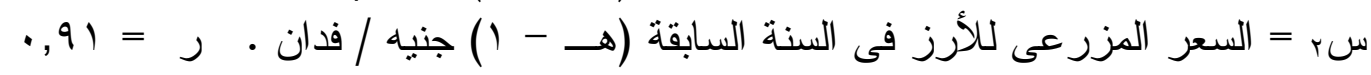

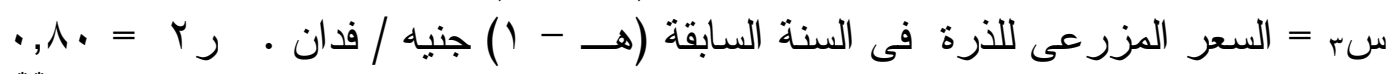

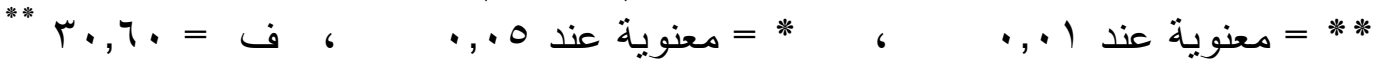

المصدر : حسبت من جدول (1) بالملحق . 


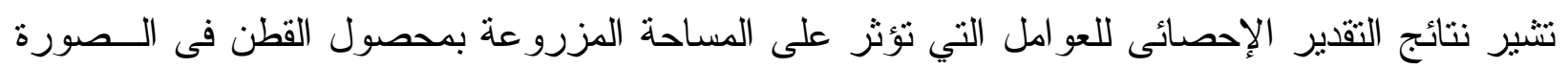

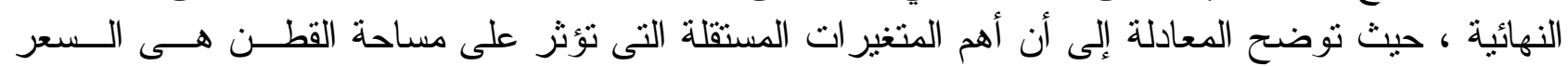

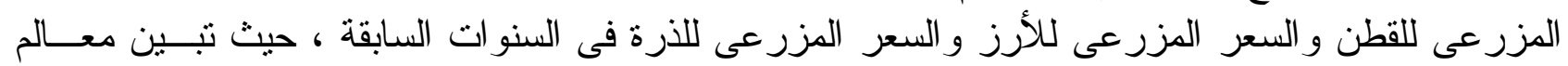

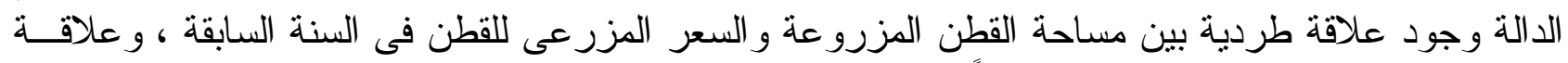

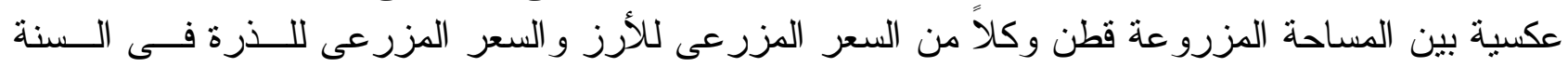

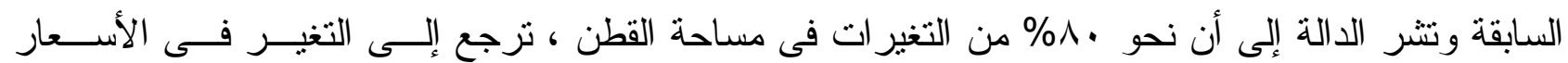

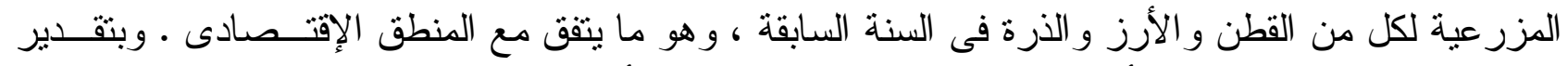

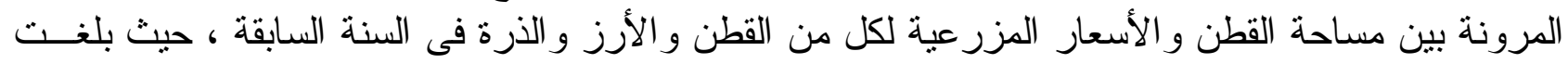

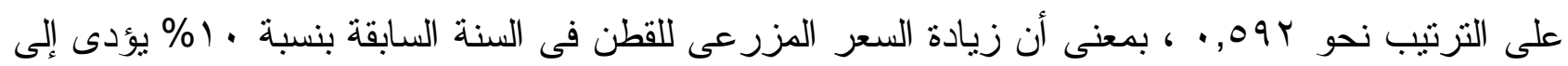

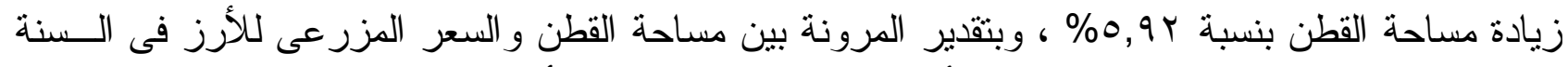

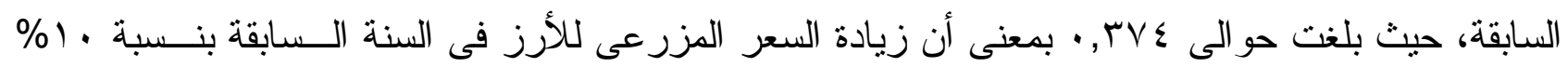

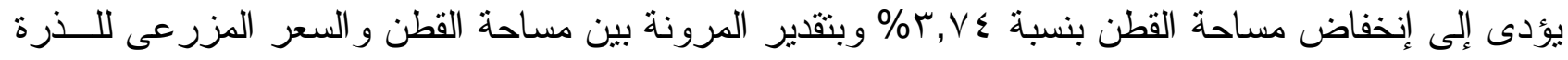

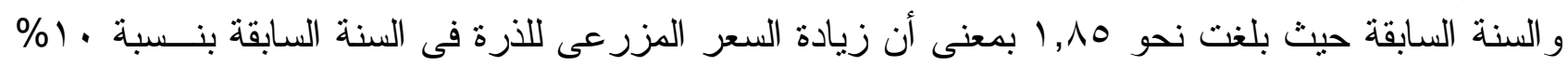

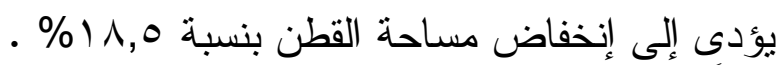

ثالثاً : العلاقة بين مساحة القطن وإنتاجيته وإنتاجية كل من الأرز والأرة الصورة العامة : الصامه :

$$
\begin{aligned}
& \text { ص ^د = } \\
& (1,1 \leq) \quad(\varepsilon, .0-) \quad(., 90) \quad \text { **** }(7,99)
\end{aligned}
$$

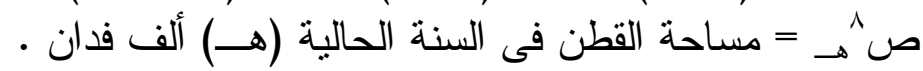

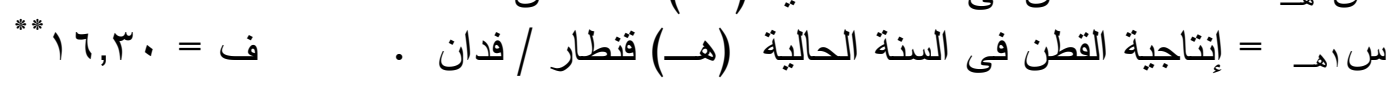

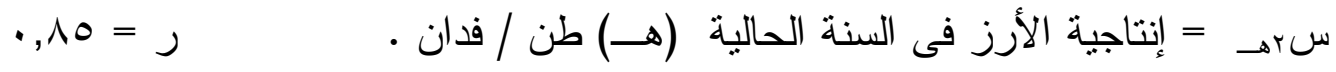

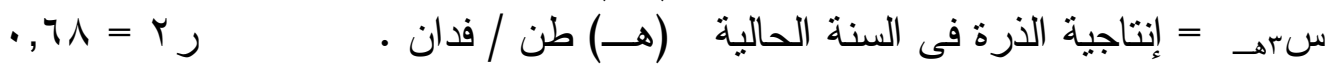

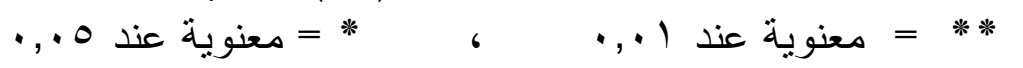

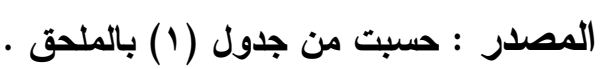

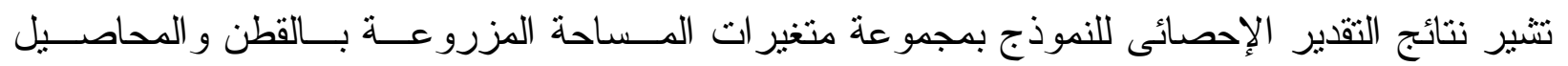

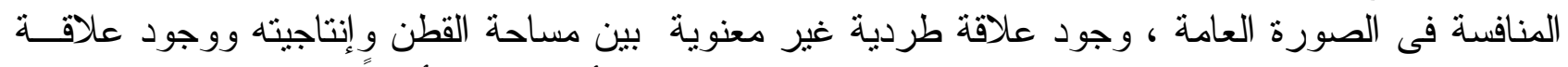

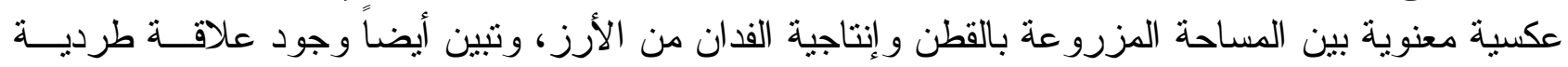

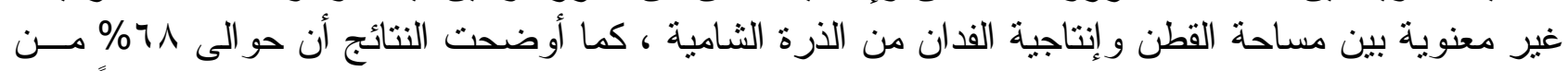

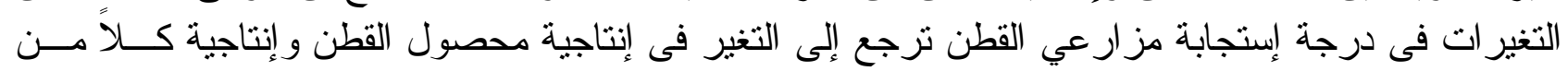

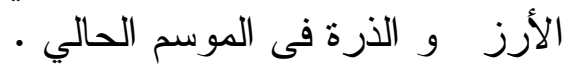

$$
\begin{aligned}
& \text { الصورة النهائية : }
\end{aligned}
$$

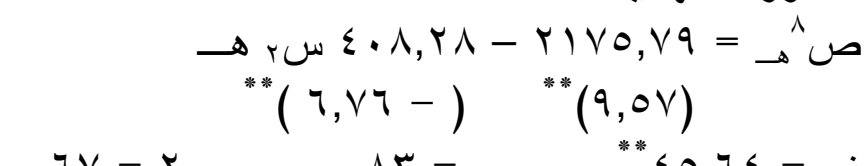

$$
\begin{aligned}
& \text { ف }
\end{aligned}
$$

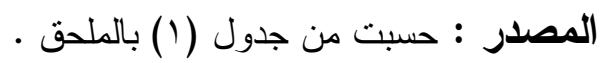

و تثنير نتائج التقدير إلى أن أهم العو امل التي تؤثز على المساحة المزروعة بمحصول القطن فى الــصورة

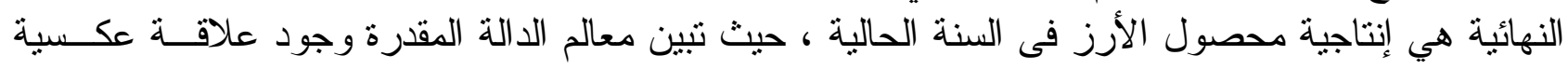

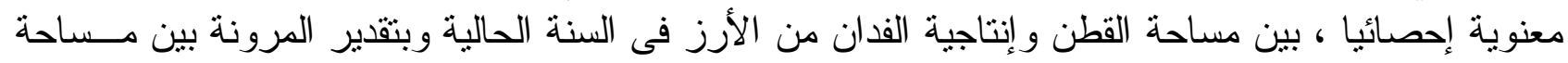

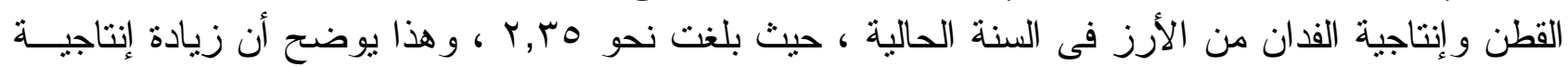

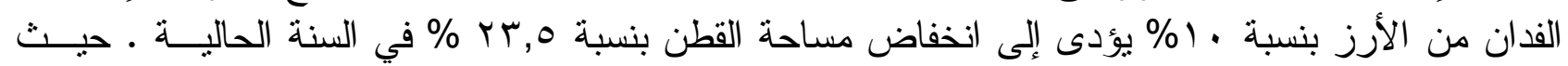




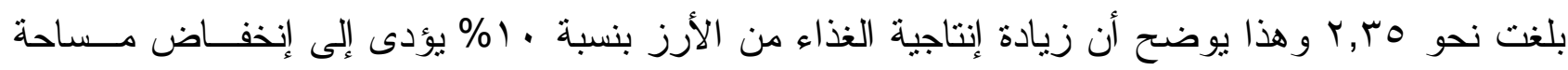

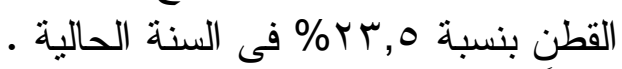
رابعاً : العلاقة بين مساحة القطن وصافى عائده وصافى العائد لكل من الأرز والأرة

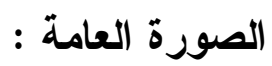

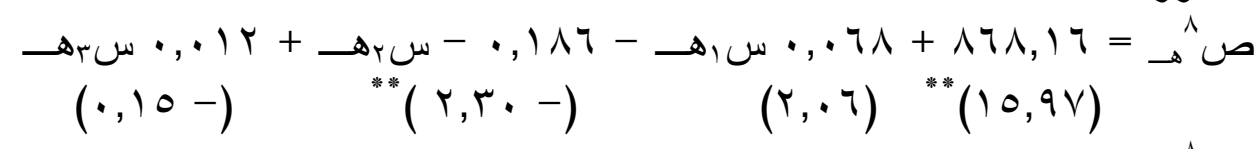

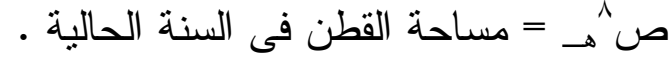

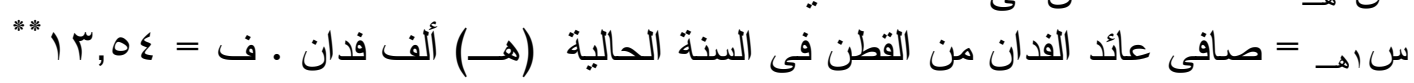

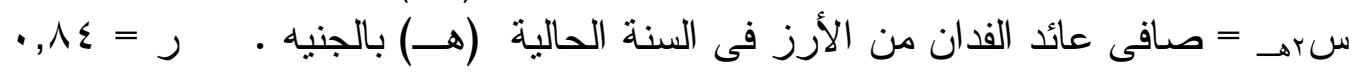

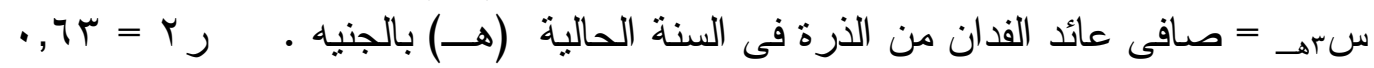

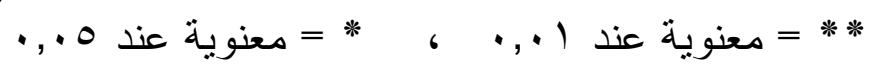

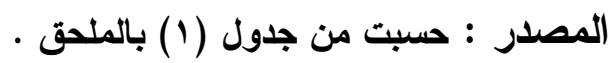

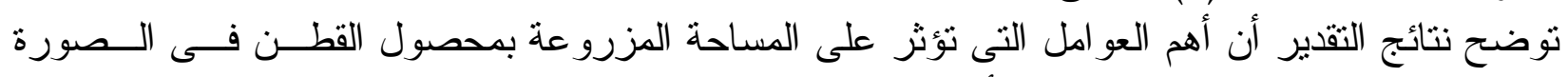

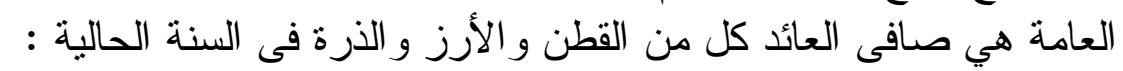
الصورة النهائية : العافية هبية

$$
\begin{aligned}
& \text { ص ^ه = } \\
& \text { *** ( }, 79-) \quad *(Y, Y T) \quad * *(17, \vee 0) \\
& \text { ف } \\
& \text { المصدر : حسبت من جدول (1) بالملحق . }
\end{aligned}
$$

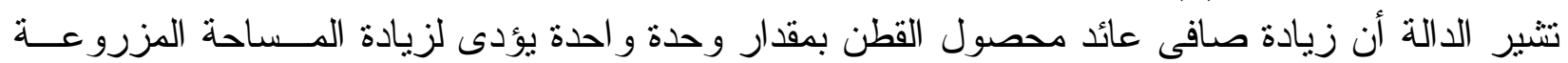

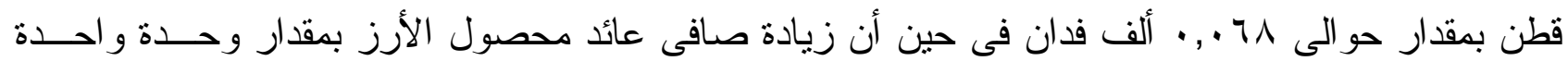

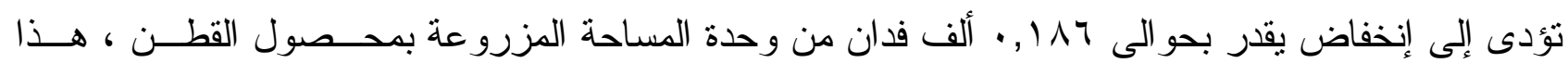
وتثير الدالة إلى أن حو الى

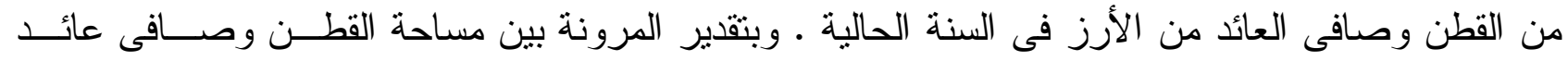

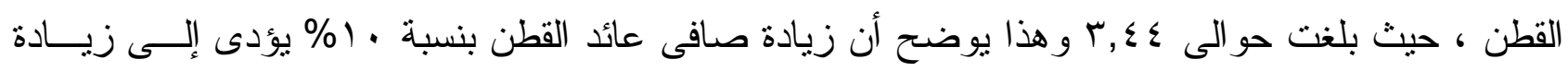

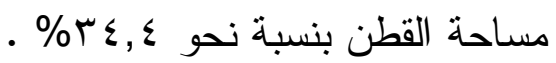

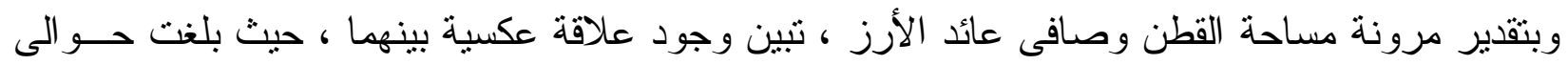

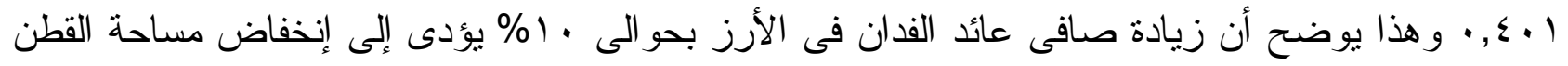

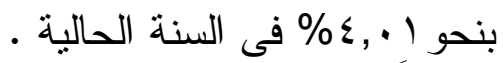

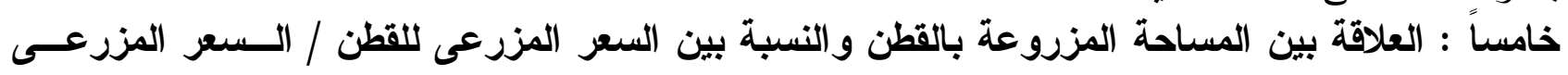

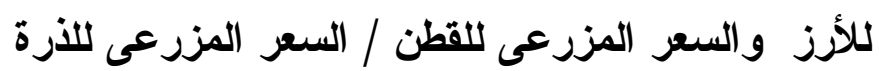
الصورة العامة : الصرز ص $(\cdot, \wedge r) \quad(1, \varepsilon \cdot) \quad(1, r)$

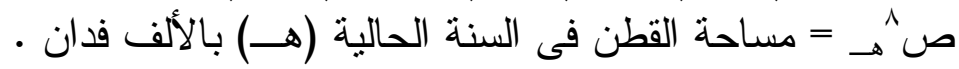

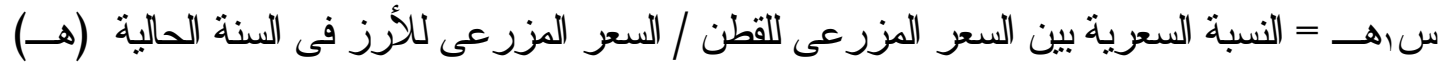

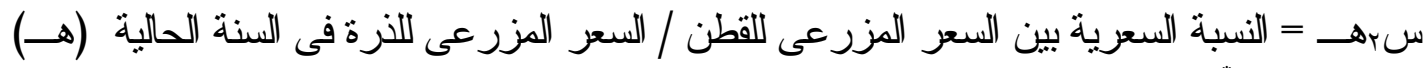
•r, $r \varepsilon=r ر$ ،

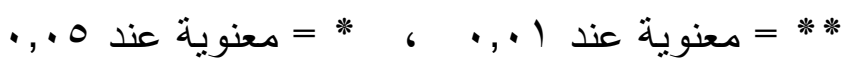

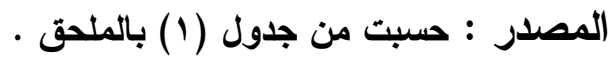




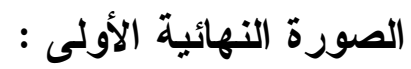

توضح تقدير الادالة فى الصورة النهائية الأولى من المعادلة الآتية :

$$
\begin{aligned}
& \text { ص ^ه = } \\
& \text { *** }(r, \wedge \wedge) \quad(\cdot, 0 Y)
\end{aligned}
$$

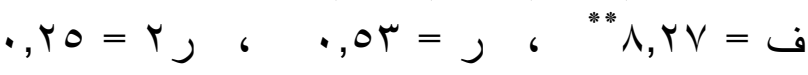

المصدر : حسبت من جدول (1) بالملحق .

تشير معالم الدالة المقدرة وجود علاقة طردية معنوية إحصائيا بين المساحة المزرو عة بالقطن و النسبة بين بالمان

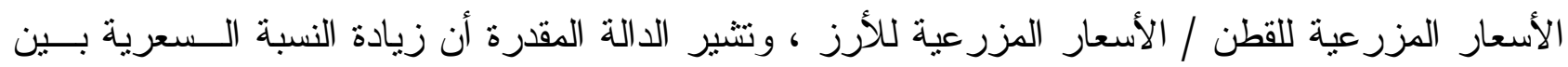

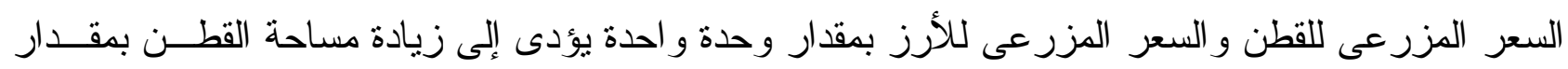

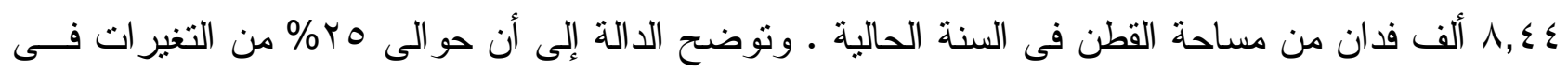

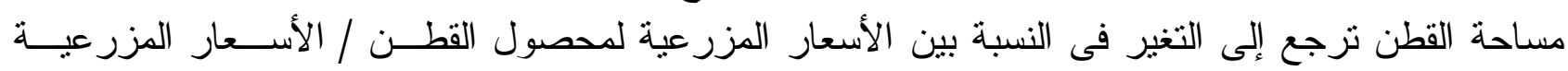

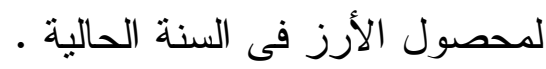

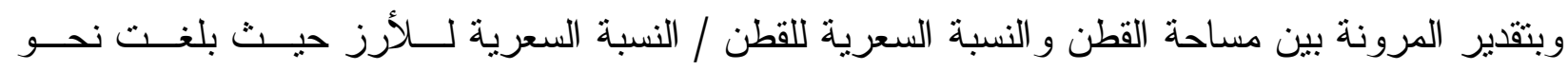

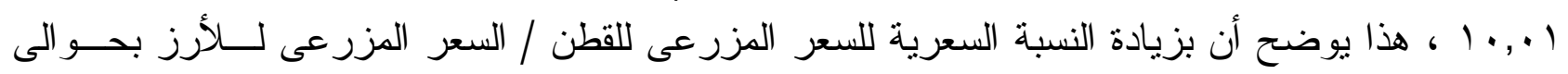

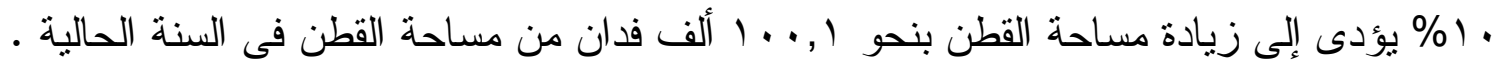
الصورة النهائية الثانية :

$$
\begin{aligned}
& \text { ص ه } \\
& \text { * }(r, O V) \quad(1,1 \wedge) \\
& \text { • }
\end{aligned}
$$

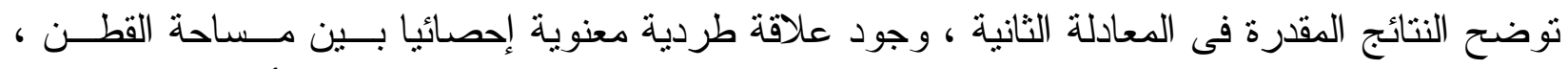

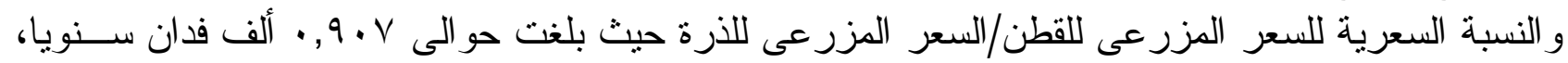

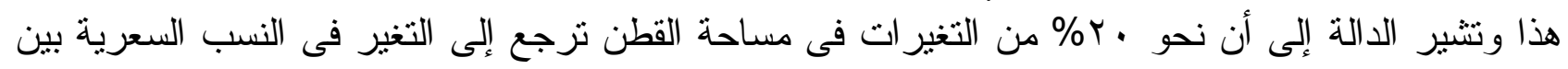

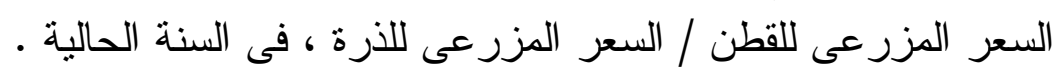

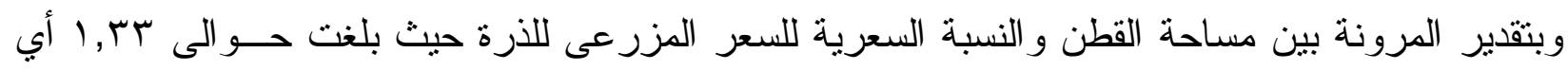

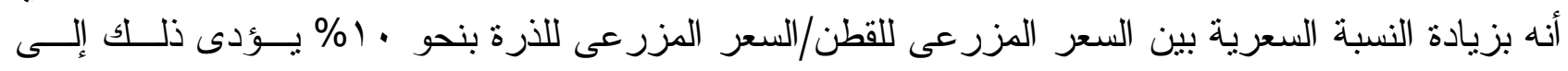

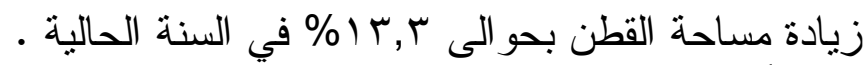

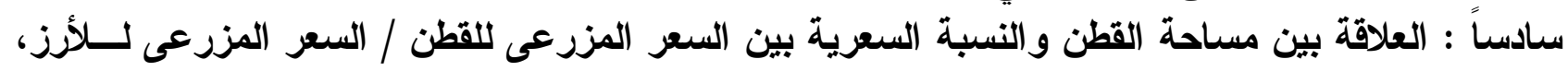

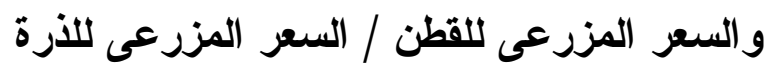

الصورة العامة : مانع

$$
\begin{aligned}
& \text { ص هـ } \\
& (1, \Sigma \wedge) \quad *(r, \cdot 1) \quad(\cdot, \circ \vee-)
\end{aligned}
$$

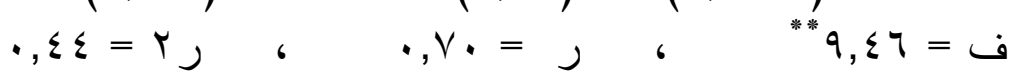

ص ^ه

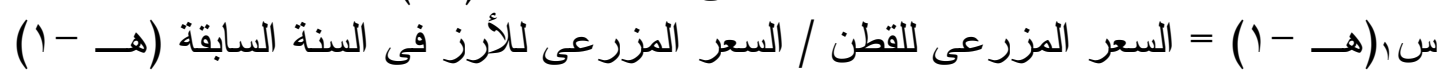

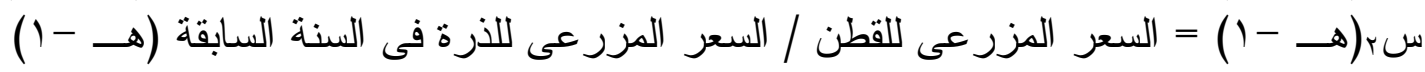

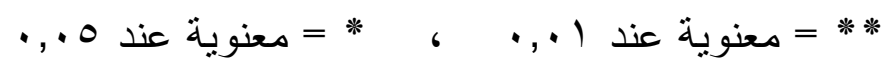

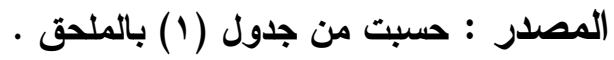




$$
\begin{aligned}
& \text { الصورة النهائية الأولى : }
\end{aligned}
$$

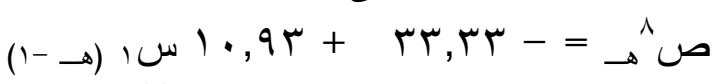

$$
\begin{aligned}
& * *(r, 9 \wedge) \quad(\cdot, 19-) \\
& \text { ف, }
\end{aligned}
$$

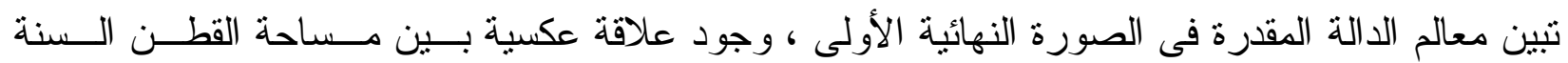

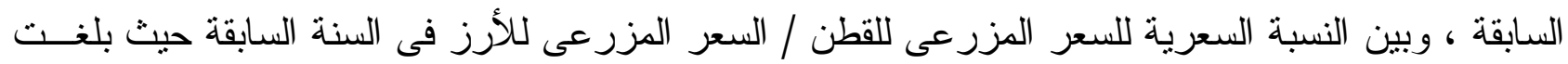

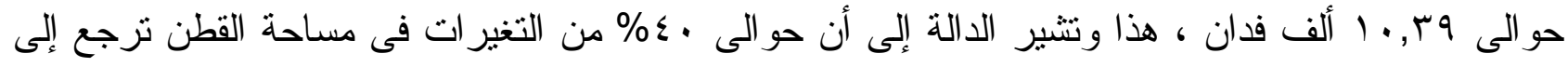

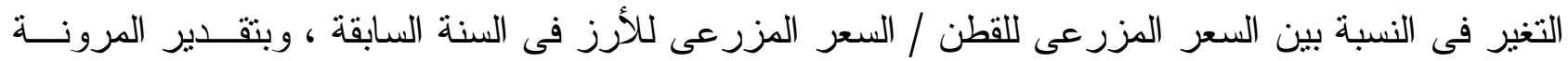
بين مساحة القطن و النسبة السعرية للسعر المزرعى للقطن / السعر المزرعى للأرز فى السنة السابقة ، حيث

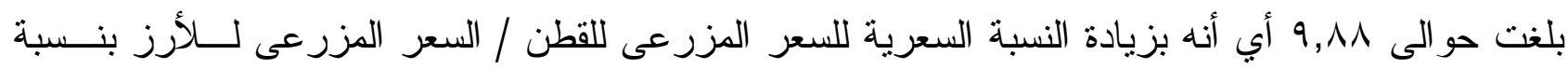

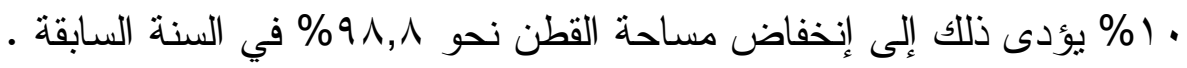

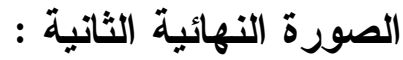

$$
\begin{aligned}
& \text { ص ده = } \\
& *(r, T) \quad(\cdot, 0 r) \\
& \text { ف }
\end{aligned}
$$

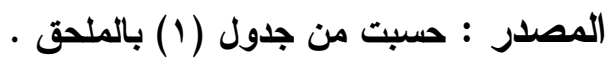

تشير معالم الدالة المقدرة فى الصورة النهائية الثانية ، وجود علاقة عكسية طردية معنوية إحصائيا ، بـين بـانية

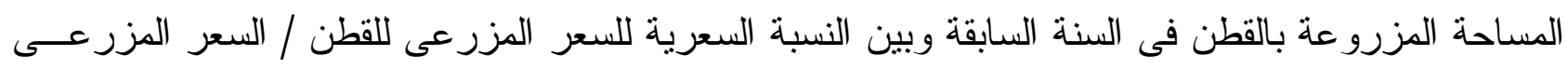

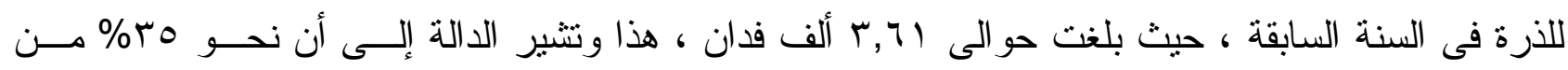

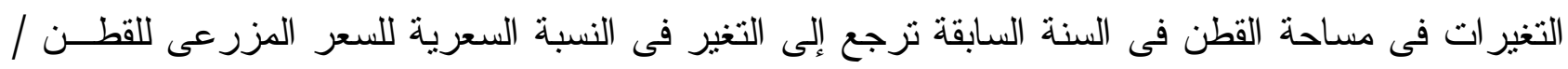

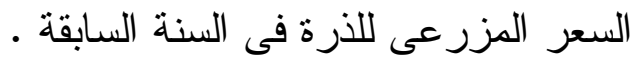

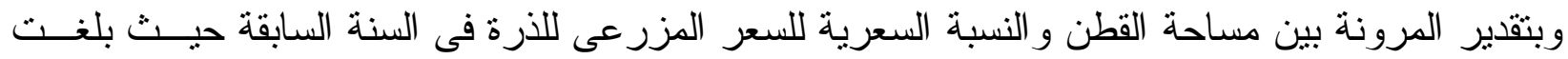

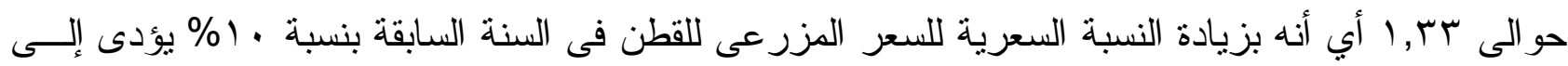

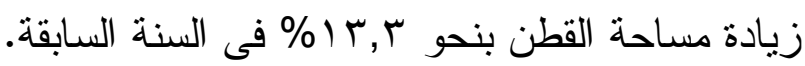

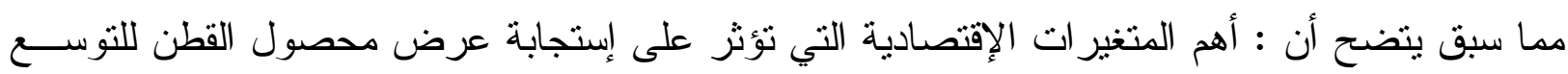

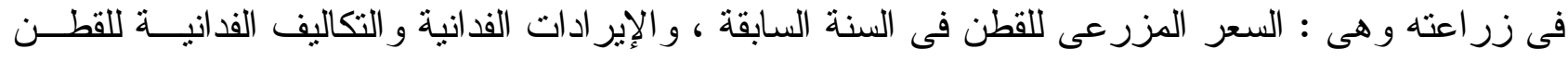

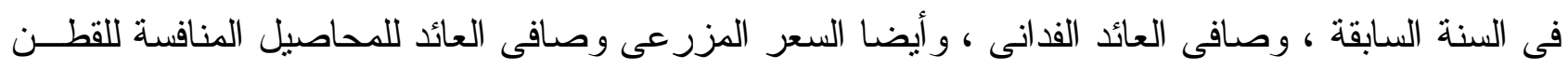
و أهمها محصولي الأرز و الذرة .

الملخص و التوصيات

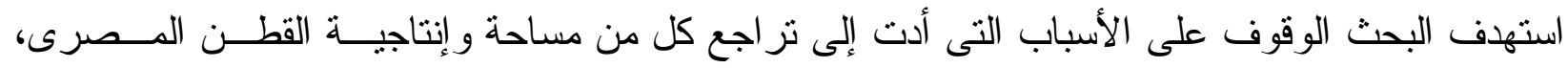

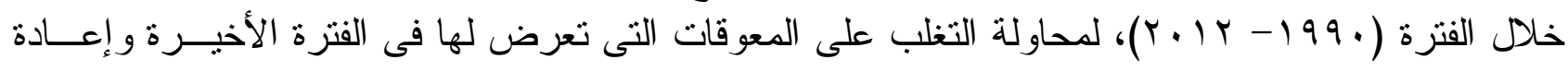

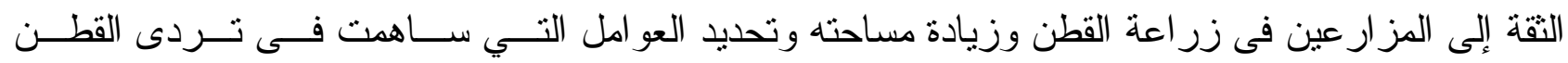

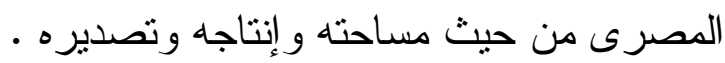

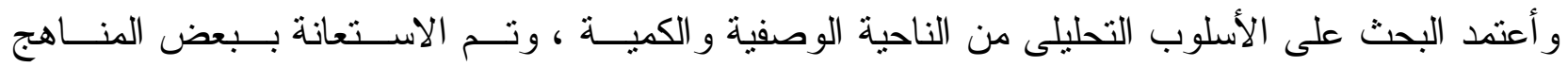

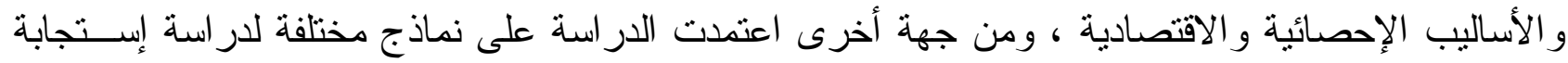
العرض لمحصول القطن للوقوف على أهم المتغيرات المؤثرة على إنتاج القطن وكذلك المحاصيل المئ المنافـسة، 


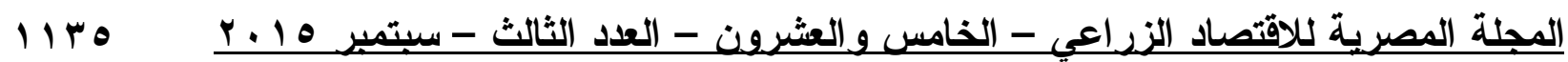

و اعتمد البحث على العديد دن المر اجع و الأبحاث و الدر اسات السابقة التى تحقق هدف الدر استة ، وتثنير أهـــم

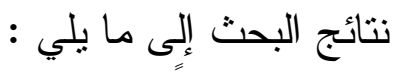

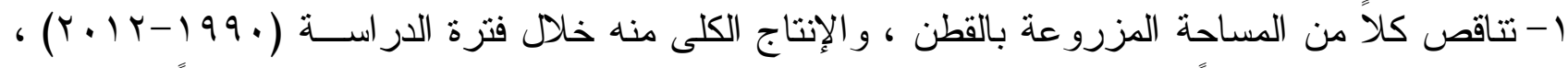

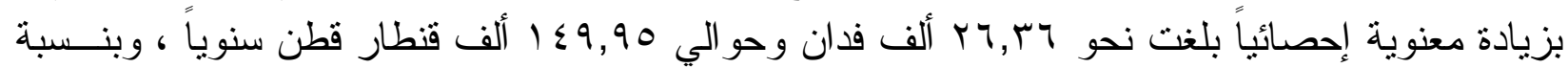

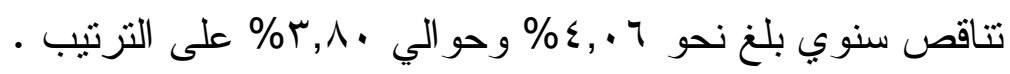

r- تز ايد الإنتاجية الفدانية من محصول القطن خلافل مدة الدر اسة و إن لم نثبت عضوية هذا التز ايد إحصائياً.

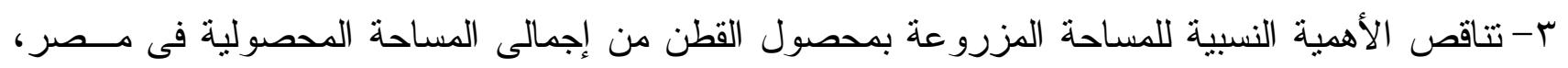

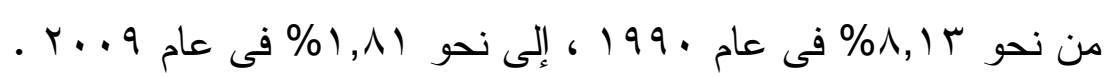

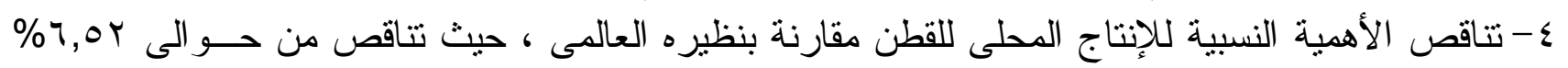

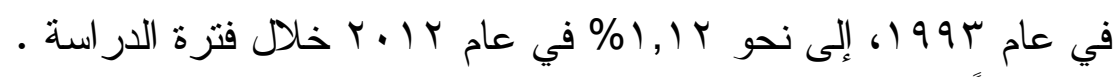

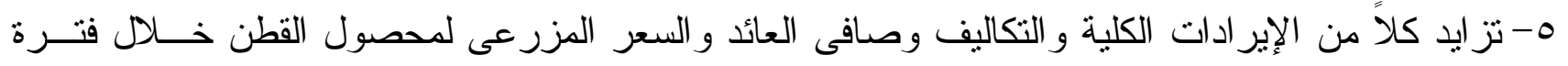

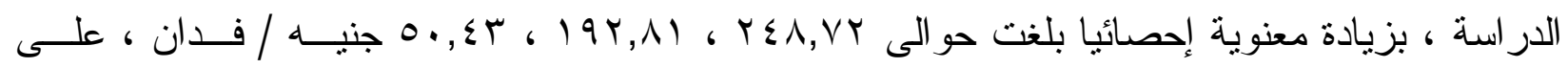

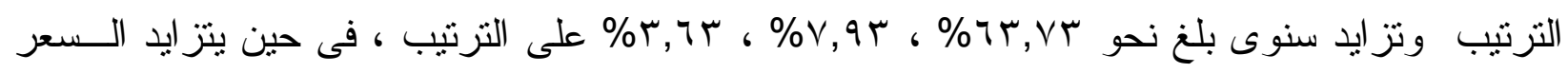

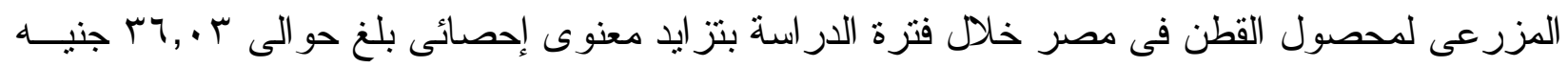

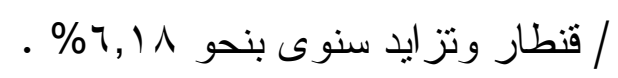

צ- تتاقص العائد المنفق على الجنيه لمحصول القطن ، خلال فترة الدر اسة حيث قدر هذا التتــاقص المعنـــى

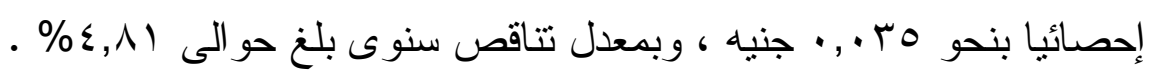

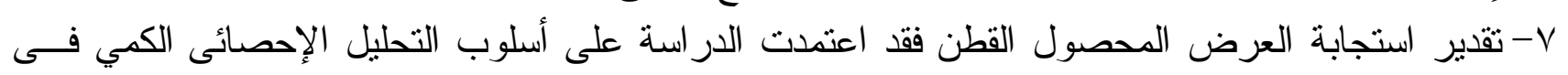

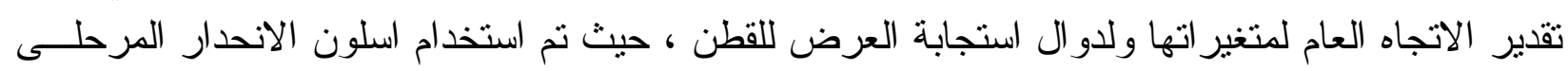

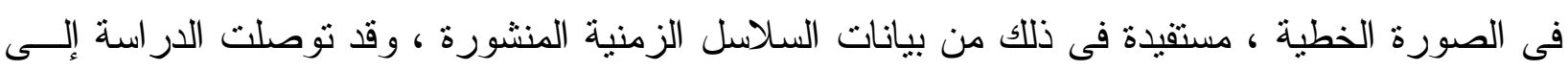

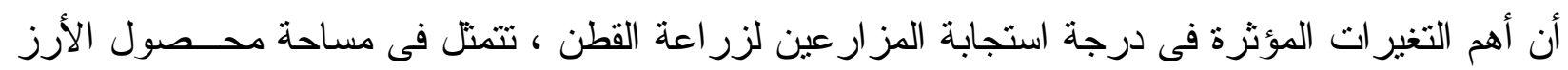

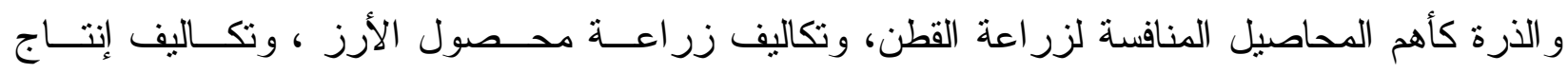

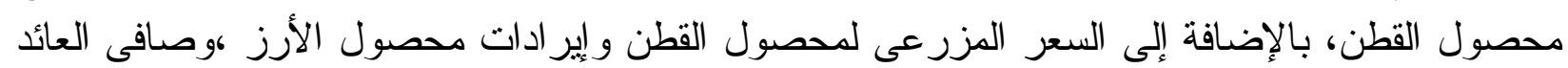

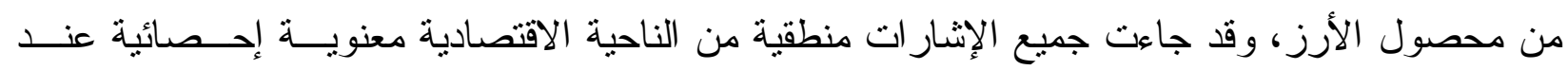

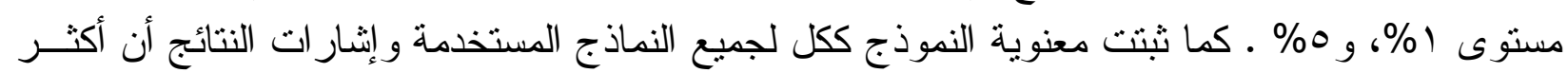

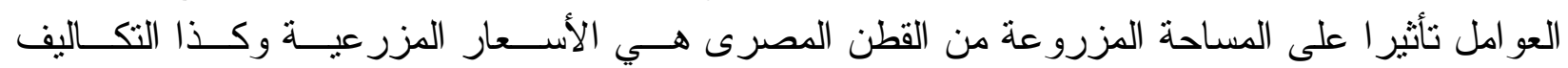
المزرعية .

ويناءا على النتائج التى توصلت إليها الاراسة فإنها توصى بما يلي :

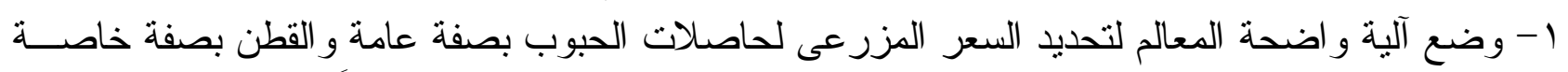

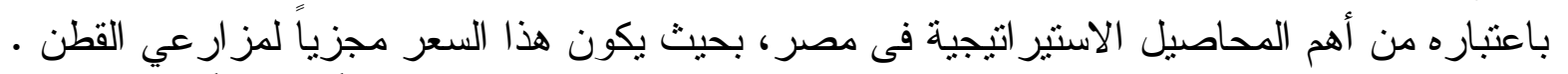

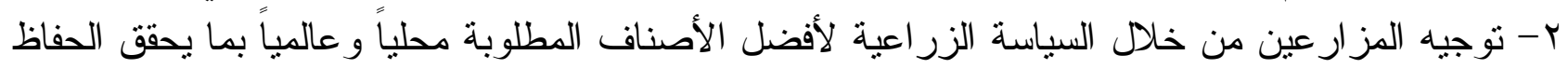

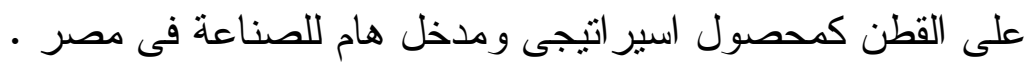

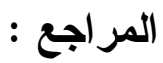

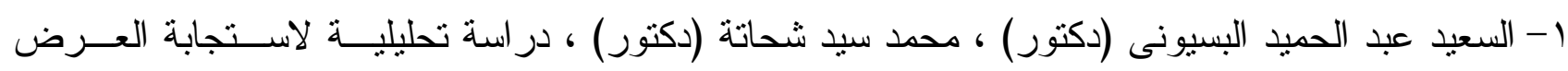
و النماذج القياسية لأهم حاصلات الخضر المصرية ، المجلة المصرية للاقتصاد الزر اعى ، المجلد السابع،

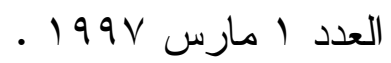




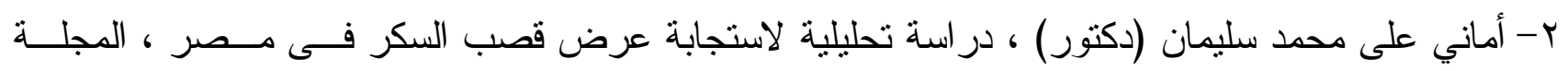

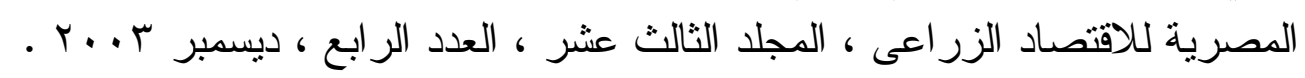

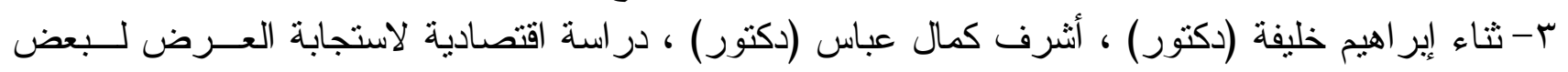
المحاصيل الزيتية فى مصر ، المجلة المصرية للاقتصاد الزر اعى ، المجلد السابع ، العدد الثاني ، سبتمبر $.199 \mathrm{~V}$

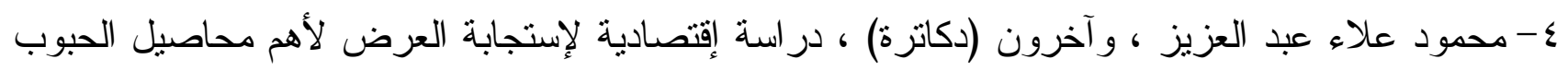

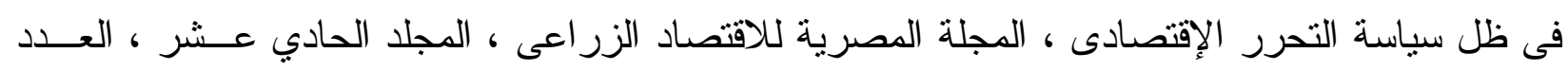

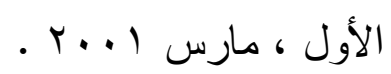

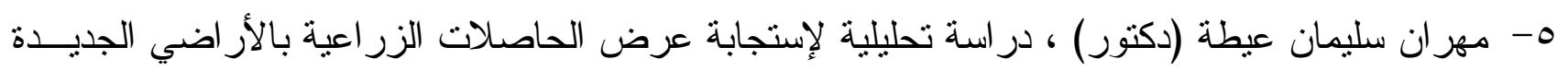

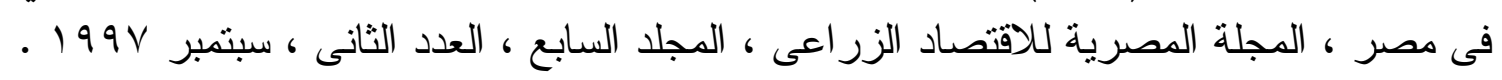

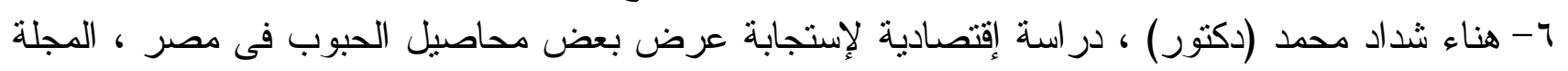

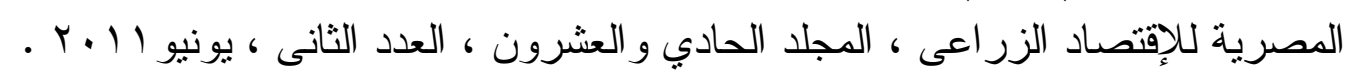

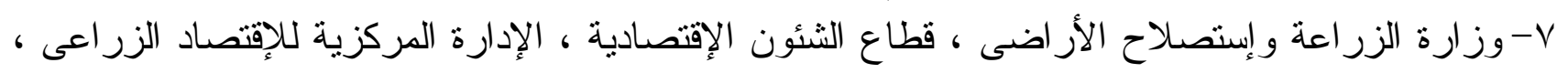
8- WWW. FAO. Org. نشرة الإقتصاد الزر اعى ، أعداد متفرقة .

9- Kautsoyiannis. A. Theohy of Econometrics. London: The Macmillum press 1 td $.2^{\text {nd }}$ Edition. 1977. 
(الملحق)

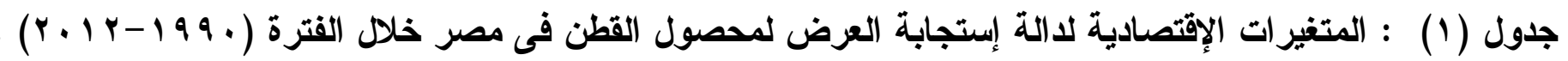

\begin{tabular}{|c|c|c|c|c|c|c|c|c|c|c|c|}
\hline الزنيه/فى للأرة & (ألف فداحة & صنيافي العئد & جنيل اللأرزعى السعر & (ألف فداحة & الفدان للقطن العائد & جنيه/فئن الكلية & الكلية للإئل ادن & جنيه/فزان اللقرعى & 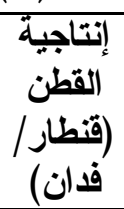 & فأفاحة & السنوات \\
\hline 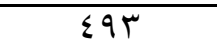 & $T \cdot \Lambda \varepsilon$ & $09 \varepsilon$ & $r \varepsilon \varepsilon$ & 1.47 & $7 \varepsilon, 0 \leqslant$ & VAr,q & $1 \leqslant Y \leqslant, \varepsilon \leqslant$ & $r \neg r, V$. & $0, Y$ & $994, .0$ & 199. \\
\hline$\leqslant \leqslant 9$ & Y107 & $7 \Sigma 1$ & rTV & 11.1 & $1 \cdot \leqslant q, \Gamma q$ & NVq & $19 r \wedge, r^{\prime}$ & Tा7,7. & 0,9 . & $\Lambda 01, Y \wedge$ & 1991 \\
\hline$\varepsilon \cdot r$ & $r \cdot \varepsilon r$ & 519 & $\sum \Gamma$ & $1 Y 10$ & $|\vee \neg \wedge$,$| .$ & $991, Y$. & rVoq,r. & $r V V, V T$ & $v, 10$ & $\Lambda \varepsilon \cdot, q_{q}$ & 1994 \\
\hline TYT & T.OA & 771 & $\{01$ & IYAY & $107 Y, V$ & I & $r q \leqslant \wedge, q$. & $r V, I r$ & $V, V \wedge$ & $\Lambda \wedge \varepsilon, \Gamma)$ & 1994 \\
\hline TY. & $r) \leqslant 7$ & $0 \wedge \varepsilon$ & $0 . \Sigma$ & $1 \mathrm{TVA}$ & $T I 1, Y$. & $1 \varepsilon \cdots, \varepsilon$. & $r \cdot 11, \tau$. & Tro,T\& & $7, \cdots$ & $V Y I, \varepsilon \varepsilon$ & $199 \leq$ \\
\hline ro7 & 1801 & $1 \cdot V Y$ & 707 & $1 \varepsilon \ldots$ & $1 \vee Y 9,0$. & $1 \leq 0$. & $r \mid V q, 0 r$ & $0 \leqslant r, V 0$ & $0, V Y$ & $V) \cdot, Y$ & 1990 \\
\hline $0 \leqslant r$ & 1871 & TYYV & $V \cdot Y$ & $1 \leqslant .0$ & $I \vee \wedge \wedge, 7$ & $1 \cdot T r, Y$ & rrvq,q. & $011, \varepsilon$ & $7, Y 7$ & $94 \cdot, 91$ & 1999 \\
\hline Vol & 1747 & $|r|$. & $\vee \wedge$ & 100. & ITVY,1. & $17 r 7,1$ & $r Y q \wedge, Y Y$ & $\varepsilon \vee \mu, \cdot$ & $7, \wedge$. & $109, Y 4$ & $199 V$ \\
\hline$\leqslant 9 \leqslant$ & $T \cdot \Lambda V$ & 1.10 & $V Y \Sigma$ & IYTY & $197, r$ & 1070,1 & $|\wedge \varepsilon Y, \wedge|$ & $r \leqslant \wedge, q$ & $0, .0$ & $\vee \wedge \wedge, \wedge)$ & 1991 \\
\hline$V \leqslant r$ & $19 \leq 0$ & 1.91 & $V T$. & 107. & $T V \varepsilon, V$ & $1 \wedge \varepsilon \Gamma, V$ & $r \backslash \vee \wedge, r$. & $r \leqslant q, \cdot$ & $7, \cdot V$ & $T \leqslant 0, \varepsilon Y$ & 1999 \\
\hline VIT & $r \ldots T$ & 710 & $0 \wedge \Gamma$ & $10 \%$ & rYI,I & $r .0 Y, q$ & $r \leqslant r \neg, V Y$ & ro., & $7, \vee \wedge$ & $01 \Lambda, T^{\prime}$ & $r \ldots$ \\
\hline VOY & $r \cdot V \wedge$ & $V \cdot 9$ & $09 Y$ & $1 \pi \leqslant 1$ & $\varepsilon \varepsilon \varepsilon, \wedge$ & $r \cdot T \Lambda, r$ & roV^,q. & $r \leqslant \vee, \cdot$ & $V, Y T$ & Vru, I & $r \ldots 1$ \\
\hline 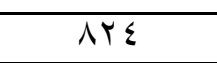 & $110 \leqslant$ & $9 \wedge r$ & $7 V 1$ & $10 \leqslant 1$ & $V \leq 7$ & $r . T r$ & $r q . v, \ldots$ & $\varepsilon 1 \cdot, \cdot$ & 7,10 & $V \cdot 7, \xi)$ & $r \ldots r$ \\
\hline 100 & 1910 & $r \| T$ & 994 & 10.9 & 1700 & 5111 & 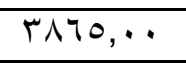 & oro,. & $V \cdot, \cdot \varepsilon$ & oro, .9 & $r \ldots r$ \\
\hline 1940 & $T \cdot M r$ & 1979 & 1.10 & $10 \mathrm{YV}$ & $r \cdot I r$ & TYVO & $\varepsilon$ $\varepsilon \uparrow, \ldots$ & 710,0 & $7,9 V$ & $V \backslash \varepsilon, V Y$ & $r \ldots \varepsilon$ \\
\hline$|A Y|$ & TYOV & $r) \leqslant 9$ & 1.79 & $1 \leqslant 7$. & $19 \leqslant Y$ & YTIV & $\varepsilon T \vee 0, \ldots$ & V77, & $T, Y Y$ & 077,01 & $r \ldots o$ \\
\hline$|\wedge \lambda|$ & 199. & $r \cdot T^{\prime}$ & $1 \cdot V V$ & 1094 & TOVT & r970 & $070 \leqslant, \cdots$ & $\vee \wedge \cdot, \cdot$ & $v, 1$. & $O V \xi, O V$ & $r \ldots r$ \\
\hline$r .01$ & 7.79 & $\Gamma \cdot \Gamma$ & $1 \leqslant 01$ & $17 V T$ & 1174 & $r \leqslant r V$ & $\varepsilon \vee \mu \neg, \cdots$ & $7 V 1, \cdot$ & $7, \wedge 7$ & or4, ร & $r \ldots v$ \\
\hline TOIY & TYYq & TYOQ & $1 \leqslant 70$ & IVV. & $1 . \wedge \mathrm{V}$ & हार. & $O r \leqslant V, \cdots$ & $\Lambda \cdot 7, \cdot$ & $7, \leqslant 7$ & $\Gamma, Y, V \lambda$ & $r \ldots \Lambda$ \\
\hline 1711 & $r T \leqslant 1$ & $r \leqslant 0 \wedge$ & $1 \leq 90$ & 1549 & rA. & r991 & $\varepsilon \varepsilon, 1, \cdots$ & $7 \vee 9, \cdot$ & $7, Y \wedge$ & $Y \wedge \varepsilon, \varepsilon r$ & $r \ldots q$ \\
\hline$r \leqslant r$. & $r r \leqslant r$ & $r \leqslant r$. & IATV & 1.94 & \&ाYฯ & EOV & 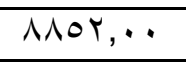 & $1 r \varepsilon \cdot, \cdot$ & $7, \leqslant 9$ & r99,1 & $r \cdot 1$. \\
\hline ro.. & 5110 & $r q \leq \varepsilon$ & 1774 & IYTI & $r .01$ & 0194 & $\wedge \Sigma \wedge \cdot, \cdots$ & $1.74,$. & $\mathrm{V}, \mathrm{VO}$ & Or.,IT & $r .11$ \\
\hline TrY. & YIOV & Tצr. & $T . T V$ & $1 \leqslant V Y$ & IYKT & $0 \leqslant 9$. & $T \bigvee / T, \cdot$ & $1179,$. & 0,09 & TrT,MT & $r \cdot I r$ \\
\hline
\end{tabular}

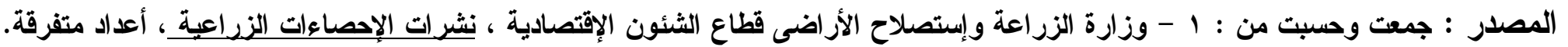

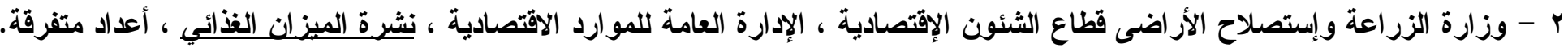




\title{
An Economic study for the Determinants of cotton cultivation in Egypt
}

\author{
Dr. Fouad Mohamed Meky Dr. Abdel Satter Mohamed Altara \\ Dr.Gamal Kotb Menacy \\ Agrie Econ . Res . Imstit _ Agric . Res . Center . Dokki . Egypt..
}

\section{Summary:}

Egyptian cotton is considered one of the most important strategic crops in Egypt. It has played a significant role in the economic history of Egypt.

The study achieved its objectives through the use of statistical analysis also. Some Researches. And previous studies also step wise which achieved the study objectives.

The findings of the study indicate that the implementation of the economic development policy of the cotton crop has a positive and negative effect on some Variables during the study period the most important of these findings is that the cultivated area of land decreased from about 993 thousand feddans in 1990 to about 333 thousand feddans in 2012.

Rice and maize are considered the most important competitive crops concerning the cultivated area .

Increasing both the total imports and the cotton cost through the study period about 248.72. 192.81. 50.43. pound and annual increase about $6.18 \%$.

Estimating a function of the supply response to the cotton crop in Egypt during the study period .

The research recommends:- that cotton finance should be available and cooperatives may play a considerable role to provide farmers by different resources at a reasonable price .

The necessity of providing data in cotton marketing and production in order to get a suitable price for the cultivator also to reduce the costs and providing the production requirements in a suitable prices . 